\title{
Minorations de hauteurs sur les variétés abéliennes
}

Bulletin de la S. M. F., tome 121, no 4 (1993), p. 509-544

<http://www.numdam.org/item?id=BSMF_1993_121_4_509_0>

(C) Bulletin de la S. M. F., 1993, tous droits réservés.

L'accès aux archives de la revue «Bulletin de la S. M. F. » (http: //smf.emath.fr/Publications/Bulletin/Presentation.html) implique l'accord avec les conditions générales d'utilisation (http://www.numdam.org/ conditions). Toute utilisation commerciale ou impression systématique est constitutive d'une infraction pénale. Toute copie ou impression de ce fichier doit contenir la présente mention de copyright.

\section{Numdam}


Bull. Soc. math. France,

121, 1993, p. 509-544.

\section{MINORATIONS DE HAUTEURS SUR LES VARIÉTÉS ABÉLIENNES}

PAR

SinNOU DAVID $(*)$

RÉSumÉ. - Nous donnons dans ce texte une minoration de la hauteur de NéronTate d'un point algébrique d'une variété abélienne principalement polarisée, définie sur $\overline{\mathbb{Q}}$. Il s'agit d'une première approche d'une généralisation à la dimension supérieure d'une conjecture de S. Lang (proposée par J. Silverman). La minoration dépend de la hauteur (stable) de la variété ainsi que d'un invariant de nature analytique. Il s'agit de la première minoration avec une borne inférieure pouvant tendre vers l'infini avec la hauteur de la variété. La preuve est basée sur une construction de transcendance.

ABstract. - In the following, we prove a lower bound for the Néron-Tate height of an algebraic point of a principally polarized abelian variety defined over $\overline{\mathbb{Q}}$. This can be seen as a first approach of a generalisation of a conjecture of S. Lang, which was suggested by J. Silverman. Our lower bound depends on the logarithmic stable height of the variety and of an analytic invariant. This is the first lower bound which can go to infinity with the height of the variety. The proof of our result is based on transcendence methods.

\section{Introduction}

Une conjecture de S. LANG propose une minoration de la hauteur de Néron-Tate d'un point $k$-rationnel d'une courbe elliptique définie sur $\overline{\mathbb{Q}}$. Cette conjecture a été partiellement démontrée par M. HindRY et J. Silverman (voir [Hi-Si1]). Nous étudions dans ce texte le cas de la dimension supérieure. La démonstration de notre résultat est basée sur des méthodes de transcendance.

Rappelons dans un premier temps la conjecture de S. Lang (voir [L, p. 92] et [Si2]) :

(*) Texte reçu le 12 mai 1992.

S. DAvid, UFR 920, Université Pierre et Marie Curie, 4, place Jussieu, 75005 Paris (France).

Classification AMS : $11 \mathrm{G}, 11 \mathrm{~J}, 14 \mathrm{~K}$.

BULLETIN DE LA SOCiÉtÉ MATHÉmATiQUE DE FRANCE 0037-9484/1993/509/\$5.00

(C) Société mathématique de France 
Conjecture 1.1 (LAng). - Pour tout corps de nombres $k$, il existe une constante positive $c(k)$ telle que pour toute courbe elliptique E définie sur $k$ et tout point $P$ d'ordre infini de $E(k)$, on ait :

$$
\widehat{h}(P) \geq c(k) \max \left\{\log \left(\left|N_{k / \mathbb{Q}}\left(\Delta_{E}\right)\right|\right), h\left(j_{E}\right)\right\},
$$

où $\widehat{h}(P)$ désigne la hauteur de Néron-Tate sur $E, N_{k / \mathbb{Q}}\left(\Delta_{E}\right)$ la norme de $k$ sur le corps des rationnels $\mathbb{Q}$ du discriminant minimal de la courbe $E$ et $h\left(j_{E}\right)$ la hauteur de Weil logarithmique et absolue de l'invariant modulaire $j_{E}$ de la courbe $E$.

Silverman [Si2, p. 396] généralise cette conjecture à la dimension supérieure. Un certain nombre de normalisations sont nécessaires pour lui donner un sens. Pour simplifier, nous nous placerons dans le cadre classique qui suit.

Soient $k$ un corps de nombres plongé dans le corps des nombres complexes $\mathbb{C}, \overline{\mathbb{Q}}$ sa clôture algébrique dans $\mathbb{C}$ et $g$ un entier $\geq 1$. On note $S_{g}$ l'espace de Siegel formé par les matrices $g \times g$, symétriques, de partie imaginaire définie positive. On notera par une apostrophe la transposée d'une matrice, et tous les vecteurs considérés seront des vecteurs lignes.

Soit $\tau \in S_{g}$; posons $\Lambda=\mathbb{Z}^{g}+\mathbb{Z}^{g} \tau$. L'espace analytique $\mathbb{C}^{g} / \Lambda$ se plonge dans un espace projectif $\mathbb{P}^{N}$ via l'application $z \mapsto \Theta_{\tau}(z)$ dont les coordonnées sont les fonctions thêta:

$$
\theta_{m}(\tau, 2 z)=\sum_{n \in \mathbb{Z}^{g}} \exp \left\{2 i \pi\left[\frac{1}{2}\left(n+m_{1}\right) \tau\left(n+m_{1}\right)^{\prime}+\left(n+m_{1}\right)\left(2 z+m_{2}\right)^{\prime}\right]\right\}
$$

où $m=\left(m_{1}, m_{2}\right)$ parcourt un système de représentants $\mathcal{Z}_{2}$ de $\left(\frac{1}{2} \mathbb{Z}^{g} / \mathbb{Z}^{g}\right)^{2}$ (sauf lorsque cela sera explicitement mentionné, on fixera dans ce texte $m_{1}$ et $m_{2}$ dans $\left[0,1\left[^{g}\right.\right.$; on notera de même $\mathcal{Z}_{\kappa}$ un système de représentants de $\left.\kappa^{-1}\left(\mathbb{Z}^{g} / \mathbb{Z}^{g}\right)^{2}\right)$. L'image de ce plongement est une variété abélienne $A(\tau)$ admettant une polarisation principale associée à la forme de Riemann $H$, où $H(z, w)=z(\operatorname{Im} \tau)^{-1} \bar{w}^{\prime}$. On notera $\mathcal{L}$ le fibré inversible ample associé à $H$. Le plongement Thêta que nous avons introduit ci-dessus est donc un plongement projectif induit par le fibré inversible très ample $\mathcal{L}^{\otimes 4}$. Si $\mathcal{M}$ est un fibré en droites sur une variété abélienne $A$, nous noterons $K(\mathcal{M})$ l'ensemble $\left\{x \in A, T_{x}^{\star}(\mathcal{M}) \simeq \mathcal{M}\right\}$. Par ailleurs, le groupe modulaire $\operatorname{Sp}_{2 g}(\mathbb{Z})$ agit sur $S_{g}$. On note $\mathcal{F}_{g}$ le «domaine fondamental» pour cette action décrit dans [Ig, p. 194]. On désigne d'autre part par $h$ la hauteur de Weil logarithmique et absolue, sur $\mathbb{P}^{N}(\overline{\mathbb{Q}})$; on notera enfin $\widehat{h}$ la partie quadratique de la hauteur de Néron-Tate associée à la forme de Riemann $H$ (qui coïncide d'ailleurs avec la hauteur de Néron-Tate, puisque $\mathcal{L}^{\otimes 4}$ est totalement symétrique). Dans tout ce qui suit - sauf mention explicite du contraire - «constante» signifiera «nombre réel $>0$ ne

TOME $121-1993-\mathrm{N}^{\circ} 4$ 
dépendant que de $g$ ». On notera $\|\cdot\|$ la norme du sup sur l'espace des matrices $g \times g$ muni de sa base canonique.

DÉfinition 1.2. - Soit $\tau$ un élément de $S_{g}$ tel que $\Theta_{\tau}(0) \in \mathbb{P}^{N}(\overline{\mathbb{Q}})$; on note $h(\tau)$, ou $h(A(\tau))$, la hauteur du point $\Theta_{\tau}(0)$ de $\mathbb{P}^{N}(\overline{\mathbb{Q}})$.

Pour toute variété abélienne principalement polarisée définie sur un corps de nombres $k$, et munie d'une structure de niveau convenable, il existe une variété abélienne de la forme $A(\tau)(\tau \in \mathcal{F})$ qui lui soit $K$-isomorphe (où $K$ est une extension finie de degré controlé de $k$ ) et telle que la hauteur naïve définie ci-dessus soit comparable à la hauteur stable $h(A)$ de $A$ (voir par exemple [Dav1, $\S 2$ ] pour cette discussion). Il sera donc suffisant pour notre étude, de nous restreindre aux variétés de type $A(\tau)$ et de travailler avec la hauteur définie ci-dessus.

La conjecture de Silverman peut dans ces conditions s'énoncer comme suit :

Conjecture 1.3 (Silverman). - Pour tout corps de nombres $k$ et tout nombre entier $g$, il existe une constante positive $c_{1}(k, g)$ telle que pour tout $\tau$ dans l'espace de Siegel $S_{g}$ tel que la variété $A(\tau)$ soit définie sur $k$, et tout point $P$ de $A=A(\tau)$, défini sur $k$, d'ordre infini modulo toute sous-variété abélienne $B \neq A$ de $A$, on ait l'inégalité :

$$
\widehat{h}(P) \geq c_{1}(k, g) h(A(\tau)) .
$$

Notons que cette conjecture, telle que nous l'avons énoncée ci-dessus est un peu plus faible que celle de Silverman, car nous n'avons pas tenu compte de la réduction additive. On pourrait également formuler la conjecture en terme de hauteur de Faltings.

Nous obtenons dans cette direction le résultat suivant :

ThÉORÈme 1.4. - Soient $g$ un entier $>0, k$ un corps de nombres et $\tau$ un élément de $\mathcal{F}$, tel que la variété abélienne $A=A(\tau)$ soit définie sur $k$. Posons :

$$
D=\max \{2,[k: \mathbb{Q}]\}, \quad h=\max \{1, h(A(\tau))\} .
$$

Il existe deux constantes $c_{1}=c_{1}(g)>0$ et $c_{2}=c_{2}(g)$ telles que tout point $P$ de $A(k)$ vérifie la propriété suivante:

- ou bien il existe une sous-variété abélienne $B$ de $A($ avec $B \neq A)$, de degré $\leq c_{2} \rho(A, k)^{g}(\log \rho(A, k))^{g}$ telle que $P$ soit d'ordre

$$
\leq c_{2} \rho(A, k)^{g}\left(\log \rho(A, k)^{g}\right) \quad \text { modulo } B,
$$

- ou bien $\widehat{h}(P) \geq c_{1}(g) \rho(A, k)^{-4 g-2}(\log 2 \rho(A, k))^{-4 g-1} h$, où $\rho(A, k)=D(h+\log D) /\|\operatorname{Im} \tau\|+D^{1 /(g+2)}$. 
On en déduit immédiatement (en utilisant la minoration triviale $\frac{1}{2} \sqrt{3} \leq\|\operatorname{Im} \tau\|$ valable pour tout $\tau \in \mathcal{F}$ ) le corollaire suivant.

Corollaire 1.5. - Pour tout entier $g>0$, il existe une constante $c_{3}$, telle que pour tout entier $D \geq 2$, tout réel $h \geq 2$, tout corps de nombres $k$ de degré $\leq D$ sur $\mathbb{Q}$ et toute variété abélienne simple $A$ définie sur $k$ et de hauteur $h(A) \leq h$, de dimension $g$, principalement polarisée, tout point $P$ de torsion (non $n u l)$ de $A(k)$ d'ordre $n$, on ait:

$$
D \geq c_{3} \frac{n^{1 /(2 g)}}{\log (n)(h+\log n)} .
$$

On notera que cette minoration est meilleure en $h$ (un $h^{-1-\varepsilon}$ au lieu d'un $h^{-3 / 2}$ ) que le résultat que nous obtenons dans [Dav1], mais elle est plus mauvaise en $n$ (un $n^{1 /(2 g)}$ au lieu d'un $n^{1 / g}$ ).

\section{Commentaires :}

- Si $A$ est une courbe elliptique, $h(A)$ est comparable à la hauteur de l'invariant modulaire $j(A)$. Par ailleurs, on déduit du $q$-développement de $j(\tau)$, les estimations suivantes, valables pour toute place à l'infini :

$$
|\log (\max \{1,|j(\tau)|\})-2 \pi| \operatorname{Im} \tau|| \leq \mathrm{O}(1)
$$

(où $\tau$ est choisi dans le domaine fondamental du demi-plan de Poincaré pour l'action de $\mathrm{SL}_{2}(\mathbb{Z})$ qui maximise sa partie imaginaire, et $\mathrm{O}(1)$ désigne une constante absolue). On en déduit que si $A$ admet bonne réduction potentielle sur $k$,

$$
\begin{aligned}
h\left(j_{A}\right) & =\frac{1}{[k: \mathbb{Q}]} \sum_{v \in S_{\infty}(k)} \varepsilon_{v} \log \left(\max \left\{1,\left|j\left(\tau_{v}\right)\right|\right\}\right) \\
& \leq 2 \pi \max \left\{\left|\operatorname{Im} \tau_{v}\right|, v \in S_{\infty}(k)\right\}+\mathrm{O}(1)
\end{aligned}
$$

(où $v$ décrit l'ensemble $S_{\infty}(k)$ des places infinies de $k$, et $\epsilon_{v}=1$ si la place $v$ est réelle et 2 si elle est complexe). Il existe donc une constante absolue $c_{4}$ telle que $\min \left\{\rho\left(A_{v}, k_{v}\right), v \mid \infty\right\} \leq c_{4} D \log (D)$ dans ce cas et par conséquent :

Corollaire 1.6. - Il existe une constante absolue $c_{5}$, telle que pour toute courbe elliptique $E$, définie sur un corps de nombres $k$ de degré au plus $D$ sur $\mathbb{Q}$, d'invariant modulaire entier, et pour tout point $P$ de $E(k)$, d'ordre infini on ait:

$$
\widehat{h}(P) \geq c_{5} D^{-6}(\log D)^{-11} h\left(j_{E}\right) .
$$

TOME $121-1993-\mathrm{N}^{\circ} 4$ 
Notons que ce corollaire découlait déjà (pour ce qui est de la dépendance en $j_{E}$ ) du premier résultat de J. Silverman (voir [Si1]) dans cette direction (qui démontrait la conjecture de S. LANG, dans le cas où la courbe $E$ n'a qu'un nombre inférieur à une constante $\alpha$ fixée de places de mauvaise réduction (multiplicative) et donc a fortiori lorsque l'invariant modulaire $j(E)$ est entier).

- Lorsque $\operatorname{dim}(A) \geq 2$, on a la majoration :

$$
\|\operatorname{Im} \tau\| \leq c_{6} D h
$$

(voir [Ma1, lemme matriciel, p. 126]). Il semble raisonnable de conjecturer :

Conjecture 1.7. - Il existe une constante $c_{7}=c_{7}(k, g)$ telle que si $\mathrm{A}$ est une variété abélienne principalement polarisée, définie sur un corps de nombres $k$, qui admet une bonne réduction potentielle sur $k$, alors:

$$
h(A) \leq c_{7} \frac{1}{[k: \mathbb{Q}]} \sum_{v \in S_{\infty}(k)}\left\|\operatorname{Im} \tau_{v}\right\| .
$$

(On choisit ici tous les $\tau_{v}$ dans $\mathcal{F}$ et si $\tau_{v}$ a plusieurs conjugués sous l'action de $\mathrm{Sp}_{2 g}(\mathbb{Z})$ dans le domaine $\mathcal{F}$, on choisit un représentant au «hasard».)

On notera que l'on ne précise pas dans cette conjecture ni la dépendance en $g$, ni la dépendance en le corps; en particulier, nous n'excluons pas une dépendance en le discriminant de $k$.

Un tel résultat, joint au THÉORÈme 1.4, donnerait une solution de la Conjecture 1.1 pour les variétés abéliennes admettant bonne réduction potentielle. On peut remarquer que $\tau_{v}$ n'est pas très bien défini dans la Conjecture 1.7 (puisqu'un élément $\tau$ de $S_{g}$ peut avoir plusieurs conjugués sous l'action de $\mathrm{Sp}_{2 g}(\mathbb{Z})$ dans le domaine $\mathcal{F}$, et que de plus, il faut tenir compte des structures de niveau et donc ne conjuguer que par le sous groupe de congruences $\langle\Gamma(4,8) \Downarrow)$. De plus, le choix de la norme $\|\cdot\|$ sur l'espace des matrices $g \times g$, influe sur la valeur de la constante $c_{7}$. Il faudrait donc faire encore un certain nombre de normalisations pour parvenir à un énoncé propre. Nous ne rentrerons pas dans ces questions au cours du présent travail.

Dans cette direction, D. Masser [Ma4] m'a indiqué qu'il savait démontrer l'inégalité :

$$
h(A) \leq c_{8}([k: \mathbb{Q}]) \sum_{v \in S_{\infty}(k)}\left\|\operatorname{Im} \tau_{v}\right\|
$$

pour une infinité de variétés abéliennes simples (sans utiliser d'hypothèse de réduction). 
Rappelons les résultats obtenus précédemment dans cette direction. On peut distinguer deux types de problèmes :

- évaluer la dépendance en le degré du corps (problèmes de type Lehmer);

- évaluer la dépendance en la variété abélienne (problèmes de type Lang évoqués ci-dessus).

Bien entendu, les résultats connus sont bien plus fins lorsque $A$ est une courbe elliptique. Pour traiter du problème de Lehmer, il est préférable de faire la distinction suivante : soient $k$ le corps de définition de $E$ et $d$ le degré de $k$ sur $\mathbb{Q}$. Si $P$ est un point d'ordre infini de $E(\overline{\mathbb{Q}})$, on note $K=k(P)$ et $D$ le degré de $K$ sur $k$. La variable intéressante pour le problème de Lehmer est $D$ et non $d D$ (puisque $E$ est fixe).

Avec ces notations, D. MAsser (voir [Ma3]) a obtenu :

$$
\widehat{h}(P) \gg_{d} D^{-3} h^{-1}(h+\log D)^{-2} .
$$

J. Silverman obtient (voir [Si1]) :

$$
\widehat{h}(P) \gg f(D, S) \log \left(\left|N_{k / \mathbb{Q}}\left(\Delta_{E}\right)\right|\right) .
$$

Ici, $S$ est le nombre de places de $k$ où $E$ admet mauvaise réduction multiplicative plus le degré de $k, \Delta_{E}$ est le discriminant minimal de $E_{/ k}$, et $f$ est une fonction décroissant exponentiellement avec $S$ et $D$.

Dans le cas de la multiplication complexe, M. LAurent (voir [Lau]) obtient une très bonne minoration en fonction du degré, qui répond à la question de Lehmer «à $\varepsilon$-près 》:

$$
\widehat{h}(P) \gg_{E} D^{-1}\left(\frac{\log D}{\log \log D}\right)^{-3} .
$$

Rappelons enfin le résultat de M. Hindry et J. Silverman (voir [Hi-Si1]) :

$$
\widehat{h}(P) \gg f\left(\sigma_{E}, D\right) \max \left\{\log \left(\left|N_{k / \mathbb{Q}} \Delta_{E}\right|\right), h\left(j_{E}\right)\right\} .
$$

Ici, $\sigma_{E}$ est le «quotient de Szpiro » de $E$, i.e.

$$
\sigma_{E}=\frac{\log \left(\left|N_{k / \mathbb{Q}} \Delta_{E}\right|\right)}{\log \left(\left|N_{k / \mathbb{Q}} N_{E}\right|\right)},
$$

$N_{E}$ étant le conducteur de $E$, et $f$ décroît exponentiellement avec $D$ et $\sigma_{E}$. En particulier, la conjecture suivante de SzPIRo [Sz, conjecture 1 (forte)] (et même une forme faible) entraîne la conjecture de Lang :

TOME $121-1993-\mathrm{N}^{\circ} 4$ 
Conjecture 1.8. - Soit $k$ un corps de nombres. Pour tout $\varepsilon>0$, il existe une constante $c_{9}=c_{9}(k, \varepsilon)$ telle que pour toute courbe elliptique $E$ définie sur $k$,

$$
\left|N_{k / \mathbb{Q}} \Delta_{E}\right| \leq c_{9}\left|N_{k / \mathbb{Q}} N_{E}\right|^{6+\varepsilon} .
$$

Notons également que $\mathrm{Z}$. ZHANG a annoncé un raffinement du théorème de M. Hindryet J. Silverman, avec en particulier une dépendance polynômiale en $D$. Enfin, dans la direction du problème de Lehmer elliptique, M. Hindry et J. Silverman obtiennent (voir [Hi-Si2]) :

$$
\hat{h}(P) \gg_{E}(D \log (D))^{-2} \text {. }
$$

Dans le cas où $A$ est une variété de dimension supérieure, il existe beaucoup moins de résultats : D. MAsser (voir [Ma1]) obtient

$$
\widehat{h}(P) \gg_{d} h^{-(2 g+1)}
$$

pour la dépendance en la variété abélienne et (voir [Ma2])

$$
\widehat{h}(P) \gg_{A} D^{-(2 g+1)}(\log D)^{-2 g}
$$

pour la dépendance en le corps.

Il est intéressant d'essayer de comparer ces deux dernières minorations avec celles que nous obtenons, au vu de la similitude des démarches (techniques de transcendance) :

- La dépendance en le degré est meilleure dans le travail de D. MAsser : cela provient du fait que D. MASSER travaille directement sur la variété (et dispose de ce fait de l'astuce d'Anderson-Baker-Coates). De plus, bien que la dimension du groupe algébrique avec lequel il travaille soit plus grande ( $2 g$ au lieu de $g+2$ dans le présent travail), la codimension de l'espace de dérivation est également plus élevée : cela permet une minoration plus fine.

- En ce qui concerne la dépendance en la variété, la comparaison peut se faire en minorant $\|\operatorname{Im} \tau\|$ par $\frac{1}{2} \sqrt{3}$ dans notre résultat (et donc en affaiblissant la minoration), et en supposant que la variété abélienne $A$ est simple. La borne inférieure que nous obtenons ainsi est alors plus faible que celle de D. MAsser. Enfin, il est intéressant de noter que la minoration de D. MAsser est valable pour tout point d'ordre infini de $P$, alors que nous devons supposer que $P$ est d'ordre infini modulo toute sous-variété abélienne de $A$. Ceci est dû au fait que la minoration de D. Masser tend vers 0 lorsque la hauteur de $A$ tend vers l'infini, alors qu'il peut arriver que 
ce soit le contraire avec notre borne (si l'on se restreind à une famille de variétés telles que $h /\|\operatorname{Im} \tau\|$ soit majoré, la borne tend vers l'infini avec la hauteur de $A$ ). En effet, une telle borne ne peut être vraie sans hypothèse de ce type sur $A$ comme le montre l'exemple très simple suivant. Soit $E_{1}$ une courbe elliptique définie sur un corps de nombres $k$ admettant un point d'ordre infini $k$-rationnel $P$. Pour toute courbe elliptique $E_{2}$ définie sur $k,(P, 0)$ est un point $k$-rationnel de $E_{1} \times E_{2}$. On aurait alors

$$
\widehat{h}(P) \gg h\left(E_{2}\right)
$$

ce qui n'est pas possible pour $h\left(E_{2}\right)$ assez grand.

- Notre résultat n'est pas contenu dans les résultats antérieurs : il est plus précis dans l'évaluation de la dépendance en la variété abélienne et donne en particulier une solution de la conjecture de Lang pour une infinité de variétés abéliennes simples, en toutes dimensions (d'après la remarque de D. MASser, [Ma4]). De plus, la dépendance en le degré du corps de définition est beaucoup plus faible dans [Ma1], que dans [Ma2], et de même la dépendance en $h$ serait (si on l'explicitait) beaucoup plus faible dans [Ma2] que dans [Ma1].

Les points principaux qui conduisent aux résultats présentés ici sont les suivants : on commence par dualiser la situation en ne considérant pas comme dans les constructions de transcendance classiques un point $P$ dans une variété abélienne $A$ mais un groupe algébrique commutatif $\mathbb{G}$. En effet, en identifiant $A$ à sa duale $A^{\vee}$, on peut voir $P$ comme un point de $A^{\vee} \cong \operatorname{Ext}\left(A, \mathbb{G}_{m}\right)$, c'est-à-dire comme une extension $\mathbb{G}$ de $A$ par $\mathbb{G}_{m}$. La construction de transcendance permet de montrer que si $\widehat{h}(P)$ est trop petit, la suite exacte :

$$
0 \rightarrow \mathbb{G}_{m} \longrightarrow \mathbb{G} \longrightarrow A \rightarrow 0
$$

est scindée à isogénie près. Une fois ce cadre posé, c'est le choix d'une direction d'extrapolation privilégiée (donnée par la théorie de la réduction des matrices symétriques) qui permet d'obtenir une contribution positive en la norme de la matrice des périodes. Il s'agit du Lemme 2.2, utilisé au paragraphe 3.2. La croissance des fonctions abéliennes est en effet «ralentie» le long de cette direction (en $R^{2} /\|\operatorname{Im} \tau\|$ ). Un principe des tiroirs est ensuite nécessaire pour ramener un «logarithme»d'un multiple de $P$ près de l'origine (cette étape est assez classique) mais, comme on n'extrapole que sur une direction, il suffit de rendre «petite» une seule coordonnée de $« \log (P) »$ (voir $\S 3.1$ ), ce qui est moins classique. Le dernier point est développé au paragraphe 2.1. Il s'agit d'estimer la hauteur de

$$
\text { TOME } 121-1993-\mathrm{N}^{\circ} 4
$$


l'analogue abélien de $\zeta(n z)-n \zeta(z)$. En écrivant ce nombre comme un polynôme en les coordonnées de $P$ et de ses multiples jusqu'à $n P$ de faible degré (au lieu de l'écrire comme un polynôme de degré $n^{2}$ en les coordonnées de $P$, on gagne un facteur $n^{2} h(A)$, ce qui est crucial pour la preuve (voir Proposition 2.8).

Nous tenons à remercier D. MASsER, dont les nombreux commentaires sur une version initiale de ce texte ont permi d'améliorer substantiellement la rédaction de ce travail.

\section{Estimations analytiques}

Pour alléger les notations, on notera dans toute la suite du texte $Y=\|\operatorname{Im} \tau\|$ (pour tout $\tau \in S_{g}$ ).

Lemme 2.1. - Il existe une constante $c_{10}=c_{10}(g)>0$, telle que la propriété suivante soit satisfaite pour tout élément $\tau$ de $\mathcal{F}$ et tout $z \in \mathbb{C}^{g}$ :

$$
\left|\log \left(\max \left\{\theta_{m}(\tau, 2 z) ; m \in \mathcal{Z}_{2}\right\}\right)-4 \pi H(\operatorname{Im} z, \operatorname{Im} z)\right| \leq c_{10} Y
$$

Voir [Dav1, th. 3-1].

Soit $e_{g}$ le $g$-ième vecteur de la base canonique de $\mathbb{C}^{g}$.

LEMme 2.2. - Il existe deux constantes

$$
c_{11}=c_{11}(g)>0, \quad c_{12}=c_{12}(g)>0
$$

telles que pour tout élément $\tau$ de $\mathcal{F}$ et tout élément $x$ de $\mathbb{R}$, on ait :

$$
c_{11} Y^{-1} x^{2} \leq H\left(x e_{g}, x e_{g}\right) \leq c_{12} Y^{-1} x^{2} .
$$

Démonstration. - Par définition, $H\left(x e_{g}, x e_{g}\right)=x e_{g}(\operatorname{Im} \tau)^{-1}\left(x e_{g}\right)^{\prime}$. C'est-à-dire, si $\alpha_{g, g}$ désigne le dernier terme diagonal de $(\operatorname{Im} \tau)^{-1}$, $H\left(x e_{g}, x e_{g}\right)=x^{2} \alpha_{g, g}$. Il s'agit donc d'estimer la valeur de $\alpha_{g, g}$. Par définition,

$$
\alpha_{g, g}=\frac{\operatorname{det}\left(\operatorname{Im} \tau_{g-1}\right)}{\operatorname{det}(\operatorname{Im} \tau)},
$$

où $\tau_{g-1}$ désigne le $(g-1)$-ième mineur principal de $\tau$. La définition du domaine fondamental $\mathcal{F}$ de l'espace de Siegel nous assure que si $\tau$ est un élément de $\mathcal{F}$, l'élément $\tau_{g-1}$ appartient à $\mathcal{F}_{g-1}$; en effet, il est clair que $\operatorname{Re} \tau_{g-1}$ n'a que des coefficients de valeur absolue $\leq \frac{1}{2}$, que $\operatorname{Im} \tau_{g-1}$ 
est encore «Minkowski réduite » (voir [Ig, p. 191]). Il suffit donc de vérifier que $\left|\operatorname{det}\left(\gamma \tau_{g-1}+\delta\right)\right| \geq 1$ pour tout $\sigma=\left(\begin{array}{cc}\alpha & \beta \\ \gamma & \delta\end{array}\right) \in \operatorname{Sp}_{2 g-2}(\mathcal{Z})$. Posons :

$$
\sigma^{\prime}=\left(\begin{array}{llll}
\alpha & 0 & \beta & 0 \\
0 & 1 & 0 & 0 \\
\gamma & 0 & \delta & 0 \\
0 & 0 & 0 & 1
\end{array}\right)
$$

Il est clair que $\sigma^{\prime} \in \operatorname{Sp}_{2 g}(\mathcal{Z})$. Comme

$$
\left|\operatorname{det}\left(\gamma \tau_{g-1}+\delta\right)\right|=\left|\operatorname{det}\left\{\left(\begin{array}{ll}
\gamma & 0 \\
0 & 0
\end{array}\right) \tau+\left(\begin{array}{ll}
\delta & 0 \\
0 & 1
\end{array}\right)\right\}\right| \geq 1,
$$

on a bien $\tau_{g-1} \in \mathcal{F}_{g-1}$. La théorie de la réduction des matrices symétriques (voir [Ig, th. 5, p. 192]) nous assure alors (pour tout $g \geq 1$ ) de l'existence d'une constante $c_{13}(g)$ telle que pour tout élément $\tau$ de $\mathcal{F}$ on ait :

$$
c_{13}(g) \prod_{i=1}^{i=g} y_{i} \leq \operatorname{det}(\operatorname{Im} \tau) \leq \prod_{i=1}^{i=g} y_{i}
$$

(où $y_{i}$ désigne le $i$-ième terme diagonal de $\operatorname{Im} \tau$ ). On en déduit :

$$
c_{13}(g-1) y_{g}^{-1} \leq \alpha_{g, g} \leq \frac{1}{c_{13}(g)} y_{g}^{-1} .
$$

Comme dans $\mathcal{F}$, on a $Y=y_{g}$, le lemme est établi (on pourra également voir [Ma1, 4-4] pour une généralisation du LEMme 2.2).

Soient maintenant $\tau$ un élément de $\mathcal{F}$ et $A(\tau)$ la variété abélienne associée. Comme $A(\tau)$ est principalement polarisée, les classes de $\overline{\mathbb{Q}}$-isomorphismes d'extensions de $A(\tau)$ par le groupe multiplicatif $\mathbb{G}_{m}$ peuvent être identifiées à des points de $A(\tau)$. Tout point $P$ de $A(\tau)$ définit dans ces conditions une telle classe d'isomorphisme. Dans [Dav2], nous avons décrit un plongement projectif $\varphi$ et des propriétés d'opérateurs de dérivations algébriques associés à un représentant de cette classe. Nous noterons $G(P)$ cette extension. Par commodité, rappelons brièvement cette construction.

Considérons l'espace vectoriel $\mathbb{C}^{g+1}$. Soient $\tau$ un élément de $\mathcal{F}$ et $\ell$ un vecteur de $\mathbb{C}^{g}, \ell=\ell^{(1)} \tau+\ell^{(2)}$, où $\ell^{(1)}$ et $\ell^{(2)}$ sont dans $\mathbb{R}^{g}$ (il y a un choix à faire puisque tous les éléments de la forme $\ell+\omega$, où $\omega$ décrit le réseau des périodes $\Lambda$, définissent le même point sur la variété abélienne; nous supposerons, sauf mention contraire, que toutes les coordonnées de $\ell^{(1)}$ et de $\ell^{(2)}$ sont comprises entre 0 et 1$)$. Fixons un élément $\alpha \in \mathcal{Z}_{2}$ tel

TOME $121-1993-\mathrm{N}^{\circ} 4$ 
que $\left|\theta_{\alpha}(\tau, 0)\right|$ soit maximal et un élément $\beta \in \mathcal{Z}_{2}$ tel que $\left|\theta_{\beta}\left(\tau, \frac{1}{2} \ell\right)\right|$ soit maximal. A ces données, on associe une extension de $A(\tau)$ par $\mathbb{G}_{m}$ définie de la façon suivante. Notons

$$
\Lambda^{\prime}=\mathbb{Z}^{g}\left(-2 i \pi \ell^{(1)^{\prime}}, I_{g}\right) \oplus \mathbb{Z}^{g}\left(2 i \pi \ell^{(2)^{\prime}}, \tau\right) \oplus \mathbb{Z}(2 i \pi, 0, \ldots, 0) ;
$$

l'espace analytique $G(\tau, \ell)=\mathbb{C}^{g+1} / \Lambda^{\prime}$ se plonge dans un espace projectif par l'application $\varphi(\tau, \ell)$ de $G(\tau, \ell)$ dans $\mathbb{P}^{N}$, dont les coordonnées sont données par les fonctions

$$
\left\{\begin{array}{l}
\theta_{m}(\tau, 2 z), \\
F_{n}(\tau, z, \ell, t)=\frac{\theta_{\alpha}(\tau, 0)}{\theta_{\beta}\left(\tau, \frac{1}{2} \ell\right)}\left(\tau, 2 z+\frac{1}{2} \ell\right) \exp \left(2 i \pi z \ell^{(1)^{\prime}}+t\right),
\end{array}\right.
$$

où $m=\left(m_{1}, m_{2}\right), n=\left(n_{1}, n_{2}\right)$ décrivent $\mathcal{Z}_{2}$ (c'est un plongement à la Serre, la description ci-dessus est donnée à une transformation linéaire près dans [Fa-Wü, satz 3, p. 190]). Cette variété analytique définit une extension analytique et donc, de façon canonique, algébrique de $A(\tau)$ par $\mathbb{G}_{m} ;$ de plus, si $A(\tau)$ est définie sur $\overline{\mathbb{Q}}$ et si $P=\Theta_{\tau}(\ell)$ est un point algébrique de $A(\tau)$, alors $G(\tau, \ell)$ est définie sur $\overline{\mathbb{Q}}$. Il n'est pas difficile de vérifier que ce plongement est projectivement normal. Soient $\iota$ le plongement naturel de $\mathbb{G}_{m}$ dans $\mathbb{P}^{1}, \pi$ la projection $G(\tau, \ell) \rightarrow A(\tau)$, et soit $N$ le fibré $\mathcal{O}(1)$ sur $\mathbb{P}^{1}$ muni de l'action naturelle de $\mathbb{G}_{m}$. Notons $\widetilde{N}$ le faisceau induit par $N$ sur $G(\tau, \ell)$ (i.e. $\left.\widetilde{N}=G(\tau, \ell) \times{ }^{\mathbb{G}_{m}} N\right)$. Le plongement décrit ci-dessus est alors celui induit par le fibré $\mathcal{F}=\widetilde{N} \otimes \pi^{\star} \mathcal{L}^{4}$. Le théorème $3-5$ de $[\mathrm{Kn}-\mathrm{La}]$ nous assure alors que $\mathcal{F}$ est normalement engendré (puisque $N$ est normalement engendré, et que $\mathcal{L}$ est ample et élevé à une puissance $\geq 3$ ) donc en particulier que le plongement associé est projectivement normal (suivant la définition de Mumford, un faisceau inversible est dit normalement engendré s'il est très ample et si l'immersion projective complète associée est projectivement normale).

Nous décrivons maintenant les extensions par un produit de $\mathbb{G}_{m}$ : soient $r$ un entier positif et $\left(\ell_{1}, \ldots, \ell_{r}\right)$ un $r$-uplet d'éléments de $\mathbb{C}^{g}$, où $\ell_{i}=\ell_{i}^{(1)} \tau+\ell_{i}^{(2)}$, avec $\ell_{i}^{(1)}, \ell_{i}^{(2)}$ éléments de $\mathbb{R}^{g}$ et de coordonnées comprises entre 0 et 1 . Notons pour $1 \leq i \leq r, \mathbb{G}_{i}$ l'extension de $A(\tau)$ paramétrée par $\ell_{i}$ construite ci-dessus, $\mathbb{G}^{\prime}=\mathbb{G}_{1} \times \ldots \times \mathbb{G}_{r}$ et $\delta$ le plongement «diagonal » de $A(\tau)$ dans $A(\tau)^{r}$, i.e. l'application définie par :

$$
\begin{aligned}
& A \stackrel{\delta}{A} A(\tau)^{r} \\
& x \longmapsto \delta(x)=(x, \ldots, x) .
\end{aligned}
$$


Soit $\mathbb{G}$ l'image réciproque de $\mathbb{G}^{\prime}$ par $\delta$, nous avons un diagramme :

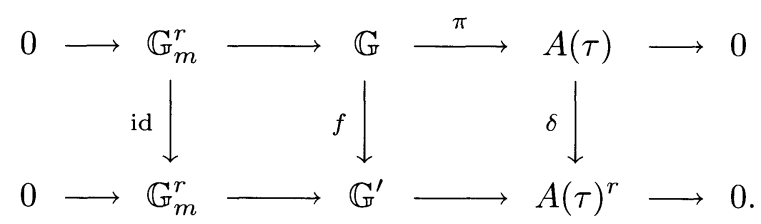

Le groupe $\mathbb{G}^{\prime}$ se plonge dans un espace projectif par un morphisme de Segre. On en déduit un plongement projectif $\varphi\left(\tau, \ell_{1}, \ldots, \ell_{r}\right)$ de $\mathbb{G}$; ce dernier est donné par les fonctions de $g+r$ variables :

$$
\prod_{i=1}^{i=r} H_{i}\left(z ; t_{i}\right)
$$

avec $H_{i}\left(z, t_{i}\right)=\theta_{m}(\tau, 2 z)$ ou $F_{n}\left(\tau, z, l_{i}, t_{i}\right)$, pour $m, n \in \mathcal{Z}_{2}$.

Nous dirons que $\mathbb{G}=\mathbb{G}\left(\tau, \ell_{1}, \ldots, \ell_{r}\right)$ est l'extension de $A(\tau)$ par $\mathbb{G}_{m}^{r}$ paramétrée par $\left(\ell_{1}, \ldots, \ell_{r}\right)$. On peut vérifier comme ci-dessus que $\mathbb{G}$ est plongé de façon projectivement normale : soit $N$ le fibré trivial sur $\left(\mathbb{P}^{1}\right)^{r}$ muni de l'action de $\mathbb{G}_{m}^{r}$ induite par le plongement naturel de $\mathbb{G}_{m}^{r}$ dans $\left(\mathbb{P}^{1}\right)^{r}$ et $\widetilde{N}$ le fibré induit par $N$ sur $\mathbb{G}$. Le plongement projectif de $\mathbb{G}$ que nous venons de décrire est défini par le fibré $\mathcal{F}$ défini cette fois par $\mathcal{F}=\widetilde{N} \otimes \pi^{\star} \mathcal{L}^{\otimes 4 r}$. Le fibré $\mathcal{F}$ est encore normalement engendré en vertu du théorème 3-5 de [Kn-La] ( $N$ est normalement engendré car très ample, [Kn-La, lemmes 6-1 et 6-2]).

DÉfinition 2.3. - Soit $\mathbb{G}=\mathbb{G}(\tau, \underline{\ell})$ l'extension de $A(\tau)$ par $\mathbb{G}_{m}^{r}$ paramétrée par les points $\left(\ell_{1}, \ldots, \ell_{r}\right)$. Supposons que $A(\tau)$ soit défini sur $\overline{\mathbb{Q}}$, de même que les points $P_{i}=\Theta_{\tau}\left(\ell_{i}\right)$ pour $1 \leq i \leq r$. Le groupe $\mathbb{G}$ est alors défini sur $\overline{\mathbb{Q}}$ et l'on pose :

$$
h\left(\tau, \ell_{1}, \ldots, \ell_{r}\right)=h(A(\tau))+\sum_{i=1}^{r} \widehat{h}\left(P_{i}\right) .
$$

Par abus de langage, nous noterons $h(\mathbb{G})$ la quantité $h\left(\tau, \ell_{1}, \ldots, \ell_{r}\right)$.

La relation (4) nous montre que pour mesurer la croissance des fonctions $H$ définissant le plongement projectif de $\mathbb{G}$, il suffit de l'étudier dans le cas particulier où $\mathbb{G}$ est une extension d'une variété abélienne par $\mathbb{G}_{m}$, c'est-à-dire qu'il suffit de majorer la croissance des fonctions $F$ introduites ci-dessus (puisque la croissance des fonctions thêta est déjà connue - c'est le Lemme 2.1). Nous avons alors :

TOME $121-1993-\mathrm{N}^{\circ} 4$ 
LEMme 2.4. - Il existe une constante $c_{14}>0$ ne dépendant que de $g$, telle que pour toute extension de $A(\tau)$ par $\mathbb{G}_{m}$ vérifiant les hypothèses ci-dessus, on ait:

$$
\log \left|F_{n}(z, t)\right| \leq c_{14}(Y+H(\operatorname{Im} z, \operatorname{Im} z)+|\operatorname{Re} t|) .
$$

Démonstration. - Immédiate à partir du Lemme 2.1. On notera que pour ce lemme, la condition $\left(\ell^{(1)}, \ell^{(2)}\right) \in[0,1]^{2 g}$ n'est pas nécessaire.

\subsection{Bases de $T_{\mathbb{G}}(\mathbb{C})$, estimations arithmétiques.}

Soit $\mathbb{G}=\mathbb{G}(\tau, \underline{\ell})$ l'extension de $A(\tau)$ par $\mathbb{G}_{m}^{r}$, paramétrée par les vecteurs $(\underline{\ell})=\left(\ell_{1}, \ldots, \ell_{r}\right)$. Par hypothèse, $T_{\mathbb{G}}(\mathbb{C})$ est identifié à $\mathbb{C}^{g+r}$ et se trouve donc muni d'une base, que nous appellerons base «naturelle».

Si $f$ est une fonction méromorphe sur $\mathbb{C}^{d+1}$, pour tout $v=\left(v_{0}, \ldots, v_{d}\right)$ dans $\mathbb{C}^{d+1}$, on note :

$$
D_{v} f=\sum_{i=0}^{d} v_{i} \frac{\partial f}{\partial z_{i}} .
$$

Quand $\left(u_{1}, \ldots, u_{n}\right)$ engendre un sous-espace vectoriel $W$ de $\mathbb{C}^{d+1}$, on dit que $f$ s'annule à un ordre $\geq T$ le long de $W$ au point $z$ si

$$
D_{u_{1}}^{t_{1}} \circ \cdots \circ D_{u_{n}}^{t_{n}} f(z)=0
$$

pour tout $t=\left(t_{1}, \ldots, t_{n}\right)$, avec $t_{i} \geq 0$ pour tout $i$, avec $1 \leq i \leq n$, tels que $|t|=\sum_{i=1}^{n} t_{i}<T$. Toutefois, pour les estimations arithmétiques, nous aurons besoin d'une autre base de l'espace tangent à l'origine de $\mathbb{G}$ que la base «naturelle». En effet, cette base est transcendante alors que $T_{\mathbb{G}}(\mathbb{C})$ possède une $\overline{\mathbb{Q}}$-structure. Pour ceci, nous relevons les dérivées de Shimura (voir [Sh]) dans le cas des extensions d'une variété abélienne afin d'obtenir une base $\mathcal{B}$ de $T_{\mathbb{G}}(\mathbb{C})$ algébrique : comme le groupe $\mathbb{G}$ est une extension d'une variété abélienne par $\mathbb{G}_{m}^{r}$, il suffit (par dévissage) de définir $\mathcal{B}$ dans le cas d'une extension d'une variété abélienne par le groupe multiplicatif. En effet, pour dériver les fonctions $H$ de la relation (4), il suffit de savoir dériver chacun des facteurs qui la composent. De plus, la matrice de passage entre la base $\mathcal{B}$ et la base «naturelle $»\left(\partial / \partial z_{1}, \ldots, \partial / \partial z_{g}, \partial / \partial t_{1}, \ldots, \partial / \partial t_{r}\right)$ se déduit aisément des matrices construites ci-dessous dans le cas particulier «intéressant» d'une extension $\mathbb{G}(\tau, \ell)$ de $A(\tau)$ par $\mathbb{G}_{m}$.

Mais, par commodité, rappelons tout d'abord la construction de Shimura. Puisque

$$
\begin{aligned}
\mathbb{C}^{g} & \longrightarrow \mathbb{P}^{N} \\
z & \longmapsto \Theta_{\tau}(z)
\end{aligned}
$$


est un plongement, on peut extraire une matrice $g \times g$ inversible de la forme

$$
\left(\begin{array}{ccc}
\frac{\partial \theta_{1}(\tau, 0)}{\partial z_{1}} & \cdots & \frac{\partial \theta_{g}(\tau, 0)}{\partial z_{1}} \\
\vdots & \vdots & \vdots \\
\frac{\partial \theta_{1}(\tau, 0)}{\partial z_{g}} & \cdots & \frac{\partial \theta_{g}(\tau, 0)}{\partial z_{g}}
\end{array}\right)
$$

de la matrice jacobienne du plongement (noter qu'a priori, il faut une matrice $(g+1) \times(g+1)$, avec une ligne de fonctions thêta parce que le plongement est projectif et non affine; la construction ci-dessus est cependant licite car les fonctions thêta sont soit paires, soit impaires, voir [Dav1, $\S 4$ ] pour plus de précisions). En choisissant un élément $\alpha \in \mathcal{Z}_{2}$ tel que $\left|\theta_{\alpha}(\tau, 0)\right|$ soit maximal, on pose

$$
P(\tau)=\theta_{\alpha}(\tau, 0)\left(\begin{array}{ccc}
\frac{\partial \theta_{1}(\tau, 0)}{\partial z_{1}} & \ldots & \frac{\partial \theta_{g}(\tau, 0)}{\partial z_{1}} \\
\vdots & \vdots & \vdots \\
\frac{\partial \theta_{1}(\tau, 0)}{\partial z_{g}} & \cdots & \frac{\partial \theta_{g}(\tau, 0)}{\partial z_{g}}
\end{array}\right)^{-1}
$$

et

$$
\left(\begin{array}{c}
\delta_{1} \\
\vdots \\
\delta_{g}
\end{array}\right)=P(\tau)\left(\begin{array}{c}
\partial / \partial z_{1} \\
\vdots \\
\partial / \partial z_{g}
\end{array}\right)
$$

Nous noterons $A_{i}$ la $i$-ième ligne de la matrice $P(\tau)$ définissant les dérivations de Shimura $\delta_{i}$. Fixons maintenant un élément $\beta$ de $\mathcal{Z}_{2}$ tel que $\left|\theta_{\beta}\left(\tau, \frac{1}{2} \ell\right)\right|$ soit maximal. Posons alors :

$$
\left(\begin{array}{c}
\delta_{1}^{\prime} \\
\vdots \\
\delta_{g}^{\prime} \\
\delta_{g+1}^{\prime}
\end{array}\right)=\left(\begin{array}{c}
\delta_{1}+\left(-2 i \pi A_{1} \cdot \ell^{\prime}-\frac{4 \delta_{1} \theta_{\beta}\left(\tau, \frac{1}{2} \ell\right)}{\theta_{\beta}\left(\tau, \frac{1}{2} \ell\right)}\right) \partial / \partial t \\
\vdots \\
\delta_{g}+\left(-2 i \pi A_{g} \cdot \ell^{\prime}-\frac{4 \delta_{g} \theta_{\beta}\left(\tau, \frac{1}{2} \ell\right)}{\theta_{\beta}\left(\tau, \frac{1}{2} \ell\right)}\right) \partial / \partial t \\
\partial / \partial t
\end{array}\right)
$$

TOME $121-1993-\mathrm{N}^{\circ} 4$ 
On pose alors $\mathcal{B}=\left\{\delta_{1}^{\prime}, \ldots, \delta_{g+1}^{\prime}\right\}$, c'est-à-dire

avec

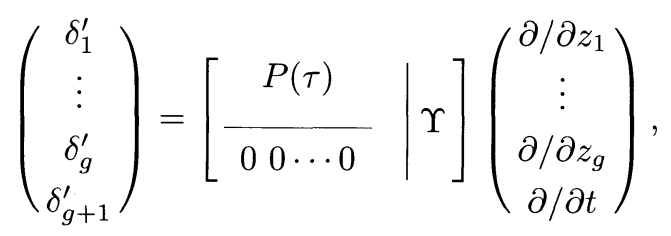

$$
\Upsilon=\left(\begin{array}{cc}
-2 i \pi A_{1} \cdot \ell^{\prime}- & \frac{4 \delta_{1} \theta_{\beta}\left(\tau, \frac{1}{2} \ell\right)}{\theta_{\beta}\left(\tau, \frac{1}{2} \ell\right)} \\
\vdots & \\
-2 i \pi A_{g} \cdot \ell^{\prime}- & \frac{4 \delta_{g} \theta_{\beta}\left(\tau, \frac{1}{2} \ell\right)}{\theta_{\beta}\left(\tau, \frac{1}{2} \ell\right)} \\
1 &
\end{array}\right)
$$

Nous noterons $Q$ la matrice de passage entre la base $\mathcal{B}$ de l'espace tangent que nous venons de construire et celle qui nous est fournie par l'identification $T_{\mathbb{G}}(\mathbb{C})=\mathbb{C}^{d}$.

Si $f$ et $g$ sont des fonctions méromorphes de $T_{\mathbb{G}}(\mathbb{C}) \rightarrow \mathbb{C}$ nous noterons $[f, g]_{i}^{\prime}$ la fonction :

$$
\delta_{i}^{\prime}(f) g-f \delta_{i}^{\prime}(g)
$$

Nous avons la proposition suivante :

Proposition 2.5. - Il existe une constante $c_{15}=c_{15}(g)>0$ vérifiant la propriété suivante : soient $h$ un nombre réel $\geq 1$ et $\tau$ un élément de $S_{g}$ tels que la variété abélienne $A(\tau)$ soit définie sur un corps de nombres $\mathbf{k}$ et vérifie $h(A(\tau)) \leq h$. Soient par ailleurs un élément $\ell$ de $\mathbb{C}^{g}$ et $\mathbb{G}$ l'extension de $A(\tau)$ par $\mathbb{G}_{m}$ définie par $\ell$. Pour tous éléments $n$ et $m$ de $\mathcal{Z}_{2}$, et tout entier $i$ compris entre 1 et $g+1$, il existe une famille de nombres algébriques $\left\{a_{\gamma, \mu, \eta}=a_{\gamma, \mu, \eta}(n, m, i, \tau) ;(\gamma, \mu, \eta) \in \mathcal{Z}_{2}{ }^{3}\right\}$ engendrant une extension de $\mathbf{k}$ de degré $\leq c_{15}$ et de hauteur $\leq c_{15} h$ telle que pour tout élément $z$ de $\mathbb{C}^{g}$, on ait:

$$
\begin{aligned}
& {\left[\theta_{m}(\tau, 2 z), F_{n}(\tau, z, \ell, t)\right]_{i}^{\prime}} \\
& \quad=\sum_{(\gamma, \mu, \eta) \in \mathcal{Z}_{2}{ }^{3}} a_{\gamma, \mu, \eta} \frac{\theta_{\gamma}\left(\tau, \frac{1}{2} \ell\right)}{\theta_{\beta}\left(\tau, \frac{1}{2} \ell\right)} \theta_{\mu}(\tau, 2 z) F_{\eta}(\tau, z, \ell, t) .
\end{aligned}
$$

Remarque. - Dans le cas où $P=\Theta_{\tau}(\ell)$ est défini sur une extension finie $\mathbf{k}^{\prime}$ de $\mathbf{k}$, on peut donc écrire :

$$
\left[\theta_{m}(\tau, 2 z), F_{n}(\tau, z, \ell, t)\right]_{i}^{\prime}=\sum_{\mu, \eta \in \mathcal{Z}_{2}} a_{\mu, \eta} \theta_{\mu}(\tau, 2 z) F_{\eta}(\tau, z, \ell, t),
$$


où les $a_{\mu, \eta}$ sont des nombres algébriques, éléments d'une extension $\mathbf{k}^{\prime \prime}$ de $\mathbf{k}^{\prime}$ de degré sur $\mathbf{k}^{\prime} \leq c_{16}$ et de hauteur $\leq c_{16} h(A(\tau))+c_{17} \widehat{h}(P)$.

Démonstration. - Il s'agit ici d'une généralisation aux extensions d'une variété abélienne par le groupe multiplicatif d'un résultat analogue obtenu précédemment pour les variétés abéliennes (voir [Dav1, th. 4-2]). Les idées principales sont les mêmes : des critères de génération normale permettent d'assurer qu'une telle relation est vraie avec des nombres complexes $a_{\gamma, \mu, \eta}$. La technique d'évaluation aux points d'ordre finis permet ensuite d'en assurer l'algébricité en les identifiant à des valeurs en $\tau$ de formes modulaires (à $q$-développement algébrique). Cet énoncé est exposé dans [Dav2, prop. 2-3-14], travail que nous comptons développer dans une publication ultérieure. Nous nous contenterons donc de donner ici quelques éléments de preuve. On commence par multiplier le membre de gauche $\operatorname{par}\left(\theta_{\beta}\left(\tau, \frac{1}{2} \ell\right)\right)^{2}$; on obtient alors :

$$
\begin{aligned}
\theta_{\beta}\left(\tau, \frac{1}{2} \ell\right)^{2}\left[\theta_{m}(\tau, 2 z), F_{n}(\tau, z, \ell, t)\right]_{i}^{\prime} & \\
=\theta_{\alpha}(\tau, 0) \exp \left(2 i \pi z \cdot \ell^{(1)^{\prime}}+t\right)\{ & \theta_{\beta}\left(\tau, \frac{1}{2} \ell\right) \theta_{n}\left(\tau, 2 z+\frac{1}{2} \ell\right) \delta_{i}\left(\theta_{m}(\tau, 2 z)\right) \\
& +4 \delta_{i}\left(\theta_{\beta}\left(\tau, \frac{1}{2} \ell\right)\right) \theta_{m}(\tau, 2 z) \theta_{n}\left(\tau, 2 z+\frac{1}{2} \ell\right) \\
& \left.-\theta_{\beta}\left(\tau, \frac{1}{2} \ell\right) \theta_{m}(\tau, 2 z) \delta_{i}\left(\theta_{n}\left(\tau, 2 z+\frac{1}{2} \ell\right)\right)\right\} .
\end{aligned}
$$

La preuve de la Proposition 2.5 se ramène donc aisément à celle du lemme suivant :

LEMME 2.6. - Il existe une constante $c_{18}=c_{18}(g)>0$ vérifiant la propriété suivante : soient $h$ un nombre réel $\geq 1$ et $\tau$ un élément de $S_{g}$ tels que la variété abélienne $A(\tau)$ soit définie sur un corps de nombres $\mathbf{k}$ et vérifie $h(A(\tau)) \leq h$. Pour tous éléments $n, m$ et $p$ de $\mathcal{Z}_{2}$, et tout entier $i$ compris entre 1 et $g$, il existe une famille de nombres algébriques $\left\{a_{\gamma, \mu, \eta}=a_{\gamma, \mu, \eta}(n, m, p, i, \tau) ;(\gamma, \mu, \eta) \in \mathcal{Z}_{2}{ }^{3}\right\}$ engendrant une extension de $\mathbf{k}$ de degré $\leq c_{18}$ et de hauteur $\leq c_{18} h$ telle que pour tout élément $(u, v)$ de $\mathbb{C}^{g} \times \mathbb{C}^{g}$, on ait :

$$
\begin{aligned}
& \theta_{m}(\tau, 2 u) \theta_{n}(\tau, 2 v) \delta_{i}\left(\theta_{p}(\tau, 2 u+2 v)\right) \\
& -\theta_{p}(\tau, 2 u+2 v) \theta_{n}(\tau, 2 v) \delta_{i}\left(\theta_{m}(\tau, 2 u)\right) \\
& \quad-\theta_{p}(\tau, 2 u+2 v) \theta_{m}(\tau, 2 u) \delta_{i}\left(\theta_{n}(\tau, 2 v)\right) \\
& =\sum_{(\gamma, \mu, \eta) \in \mathcal{Z}_{2}{ }^{3}} a_{\gamma, \mu, \eta} \theta_{\gamma}(\tau, 2 u) \theta_{\mu}(\tau, 2 v) \theta_{\eta}(\tau, 2 u+2 v) .
\end{aligned}
$$

TOME $121-1993-\mathrm{N}^{\circ} 4$ 
Démonstration. - Notons $\pi_{1}$ la projection $A \times A \rightarrow A$ sur le premier facteur, $\pi_{2}$ la projection sur le deuxième facteur et enfin $m$ l'addition :

$$
\begin{aligned}
m: A \times A & \longrightarrow A \\
(P, Q) & \longmapsto m(P, Q)=P+Q .
\end{aligned}
$$

Un calcul de facteur d'automorphie montre que le membre de gauche de l'équation du Lemme 2.6 est un élément de :

$$
\Gamma\left(A \times A, \pi_{1}^{\star} \mathcal{L}^{\otimes 4} \otimes \pi_{2}^{\star} \mathcal{L}^{\otimes 4} \otimes m^{\star} \mathcal{L}^{\otimes 4}\right),
$$

dont un système générateur est donné par les :

$$
\left(\theta_{\gamma}(\tau, 2 u) \theta_{\mu}(\tau, 2 v) \theta_{\eta}(\tau, 2 u+2 v)\right)_{\gamma, \mu, \eta \in \mathcal{Z}_{2}} .
$$

L'existence de nombres complexes $a_{\gamma, \mu, \eta}$, vérifiant la relation du Lemme 2.6 en découle. Pour vérifier l'algébricité, on utilise la technique d'évaluation aux points d'ordre fini :

Lemme 2.7. - Soient $A$ une variété abélienne, $\mathcal{L}$ un fibré ample sur $A$, engendré par ses sections. La flèche naturelle

$$
H^{0}(A, \mathcal{L}) \longrightarrow H^{0}\left(K(\mathcal{L}), \mathcal{L}_{\mid K(\mathcal{L})}\right)
$$

est injective.

Voir [M-B, lemme 2-3-1].

Il est facile de vérifier que $\mathcal{M}=\pi_{1}^{\star} \mathcal{L}^{\otimes 4} \otimes \pi_{2}^{\star} \mathcal{L}^{\otimes 4} \otimes m^{\star} \mathcal{L}^{\otimes 4}$ est engendré par ses sections et que $K(\mathcal{M}) \subset(A \times A)_{12}$ (l'ensemble des points de 12-torsion de $A \times A$; plus précisément, on trouve

$$
\left.K(\mathcal{M})=\left\{(x,-2 x+y), x \in A_{12}, y \in A_{4}\right\}\right) .
$$

Le fibré $\mathcal{M}$ est donc ample (voir [Mum, p. 84]). On peut donc résoudre le système linéaire défini par la relation du Lemme 2.6, d'inconnues $a_{\gamma, \mu, \eta}, \gamma, \mu, \eta \in \mathcal{Z}_{2}$, les équations étant données en faisant varier $(u, v)$ dans $K(\mathcal{M})$. Cette résolution permet d'exprimer les $a_{\gamma, \mu, \eta}$ comme des valeurs en $\tau$ de fonctions modulaires de poids 0 et de niveau convenable (le niveau « $\Gamma(576,1152) »$ convient), à $q$-développement algébrique (on utilise pour le membre de gauche du système les propriétés modulaires des dérivations de Shimura, résumées par exemple dans [Dav1, lemme 4-8]). La proposition 4-9 de [Dav1] permet alors de conclure que les $a_{\gamma, \mu, \eta}$ sont 
algébriques, de hauteur $\leq c_{18} h$, éléments d'une extension de $\mathbf{k}$ de degré au plus $c_{18}$. Ce qui permet d'établir le Lemme 2.6.

Pour pouvoir conclure au paragraphe 3.3, nous avons besoin de contrôler la hauteur de

$$
\frac{f(\ell)}{\theta_{\beta}\left(\tau, \frac{1}{2} \ell\right) \theta_{\beta_{N}}\left(\tau, N \frac{1}{2} \ell\right)}
$$

où $f$ est la fonction de $\mathbb{C}^{g}$ dans $\mathbb{C}$ définie par :

$$
f(\ell)=\theta_{\beta}\left(\tau, \frac{1}{2} \ell\right) \delta_{i}\left(\theta_{\beta_{N}}\left(\tau, N \frac{1}{2} \ell\right)\right)-N \theta_{\beta_{N}}\left(\tau, N \frac{1}{2} \ell\right) \delta_{i}\left(\theta_{\beta}\left(\tau, \frac{1}{2} \ell\right)\right),
$$

et où $N$ est une puissance de $2, \beta$ un élément de $\mathcal{Z}_{2}$ tel que $\left|\theta_{\beta}\left(\tau, \frac{1}{2} \ell\right)\right|$ soit maximal, $\beta_{N}$ un élément de $\mathcal{Z}_{2}$ tel que $\left|\theta_{\beta_{N}}\left(\tau, N \frac{1}{2} \ell\right)\right|$ soit maximal et $\ell$ un élément de $\mathbb{C}^{g}$ tel que $\Theta_{\tau}(\ell) \in \overline{\mathbb{Q}}$. Un calcul de facteur d'automorphie montre aisément que la fonction $f(4 \ell)$ peut être identifiée à une section de $\Gamma\left(A(\tau), \mathcal{L}^{\otimes\left(N^{2}+1\right)}\right)$. Des arguments d'évaluation aux points d'ordre finis de $A(\tau)$ et les propriétés modulaires des dérivations de Shimura permettent, en reproduisant les arguments de [Dav1, §4], de conclure que $f(\ell)$ peut s'écrire comme un polynôme de degré $N^{2}+1$ en les $\theta_{\gamma}\left(\tau, \frac{1}{2} \ell\right)$ (avec $\gamma \in \mathcal{Z}_{2}$ ), dont les coefficients sont des nombres algébriques de hauteur $\leq c_{19} h$. Malheureusement, si on applique directement l'argument ci-dessus, on est obligé d'appliquer le lemme d'évaluation avec des points de torsion d'ordre $N^{2}+1$ d'où des nombres algébriques - certes de hauteur controlée - mais de très gros degré. De plus, nous avons besoin pour nos estimations de la hauteur de Néron-Tate de $P$ et non de sa hauteur de Weil, qui est celle qui intervient naturellement dans ces estimations (la hauteur du nombre $\theta_{\gamma}\left(\tau, \frac{1}{2}\right) / \theta_{\beta}\left(\tau, \frac{1}{2} \ell\right.$ ) est controlée par la hauteur de Weil de $P$ ). On peut certes se ramener à la hauteur de Néron-Tate de $P$, grâce au théorème de comparaison de Manin-Zarhin (cf. [M-Z, th. 3-2]), mais on aurait alors

$$
h\left(\frac{f(\ell)}{\theta_{\beta}\left(\tau, \frac{1}{2} \ell\right) \theta_{\beta_{N}}\left(\tau, N \frac{1}{2} \ell\right)}\right) \leq c_{20}\left(N^{2} \widehat{h}(P)+N^{2} h\right),
$$

ce qui serait rédhibitoire (en effet, $D T N^{2} h$ est plus grand que $U$; voir le paragraphe 3 ). Nous allons donc écrire $f(\ell)$ comme un polynôme en les coordonnées de $\Theta_{\tau}\left(\frac{1}{2} \ell\right)$ et de ses multiples. Ainsi, on appliquera le lemme de comparaison de hauteur avec $|\widehat{h}(N P)-h(N P)| \ll h$, et on pourra remplacer dans la majoration $N^{2} h$ par $\log (N) h$, ce qui permet de conclure. C'est pour cette écriture qu'il est plus commode de supposer que $N$ est une puissance de 2 . Nous démontrerons l'estimation suivante :

$$
\text { TOME } 121-1993-\mathrm{N}^{\circ} 4
$$


Proposition 2.8. - Il existe une constante $c_{21}$, telle que pour tout $\tau \in \mathcal{F}$ tel que $A(\tau)$ soit définie sur un corps de nombres $\mathbf{k}$, pour tout point $P$ de $A(\mathbf{k})$, et toute puissance $N$ de 2 , le nombre

$$
\frac{f(\ell)}{\theta_{\beta}\left(\tau, \frac{1}{2} \ell\right) \theta_{\beta_{N}}\left(\tau, N \frac{1}{2} \ell\right)}
$$

soit algébrique, élément d'une extension de $\mathbf{k}$ de degré au plus $c_{21}$ et de hauteur $\leq c_{21}\left(N^{2} \widehat{h}(P)+\log (N) h\right)$. Ci-dessus, $\ell$ désigne un élément de $\mathbb{C}^{g}$ tel que $\Theta_{\tau}(\ell)=P, \beta$ un élément de $\mathcal{Z}_{2}$ tel que $\left|\theta_{\beta}\left(\tau, \frac{1}{2} \ell\right)\right|$ soit maximal, et $\beta_{N}$ un élément de $\mathcal{Z}_{2}$ tel que $\left|\theta_{\beta_{N}}\left(\tau, N \frac{1}{2} \ell\right)\right|$ soit maximal.

Démonstration. - Effectuons tout d'abord le changement de variable $\ell \mapsto 4 \ell$.

LEMme 2.9. - Il existe une constante $c_{22}$ telle que pour tout $\beta, \lambda \in \mathcal{Z}_{2}$, pour tout $i$ satisfaisant $1 \leq i \leq g$, il existe des nombres algébriques

$$
a_{\gamma, \delta}=a_{\gamma, \delta}(\beta, \lambda, i, \tau), \quad \gamma \in \mathcal{Z}_{2}, \delta \in \mathcal{Z}_{4}
$$

de hauteur $\leq c_{22} h$, engendrant une extension $K$ de $\mathbf{k}$ de degré $\leq c_{22}$ tels que :

$$
\begin{aligned}
\theta_{\beta}(\tau, 2 \ell) \delta_{i}\left(\theta_{\lambda}(\tau, 4 \ell)\right)-2 \theta_{\lambda}(\tau, 4 \ell) \delta_{i}\left(\theta_{\beta}(\tau, 2 \ell)\right) \\
=\sum_{\gamma \in \mathcal{Z}_{2}, \delta \in \mathcal{Z}_{4}} a_{\gamma, \delta} \theta_{\gamma}(\tau, 2 \ell) \theta_{\delta}(\tau, 4 \ell) .
\end{aligned}
$$

Démonstration. — Rappelons tout d'abord le critère de génération normale suivant :

Lemme 2.10. (Mumford-Koizumi). - Soient $A$ une variété abélienne et $\mathcal{L}$ un fibré ample sur $A$. Soient de plus $m$ et $n$ deux entiers, avec $n \geq 3$ et $m \geq 2$. La flèche naturelle

$$
\Gamma\left(A, \mathcal{L}^{\otimes n}\right) \otimes \Gamma\left(A, \mathcal{L}^{\otimes m}\right) \longrightarrow \Gamma\left(A, \mathcal{L}^{\otimes n+m}\right)
$$

est surjective.

Voir [Kem, th. 2].

Un calcul de facteur d'automorphie nous montre alors que le membre de gauche de l'équation (7) est un élément de $\Gamma\left(A(\tau), \mathcal{L}^{\otimes 20}\right)$. Or, le critère de génération normale de Mumford-Koizumi nous assure que la flèche naturelle :

$$
\Gamma\left(A(\tau), \mathcal{L}^{\otimes 16}\right) \otimes \Gamma\left(A(\tau), \mathcal{L}^{\otimes 4}\right) \longrightarrow \Gamma\left(A(\tau), \mathcal{L}^{\otimes 20}\right)
$$

est surjective. Les $\left(\theta_{\gamma}(\tau, 2 \ell) \theta_{\delta}(\tau, 4 \ell)\right)_{\gamma \in \mathcal{Z}_{2}, \delta \in \mathcal{Z}_{4}}$ forment donc un système générateur de $\Gamma\left(A(\tau), \mathcal{L}^{\otimes 20}\right)$. L'existence des $a_{\gamma, \delta}$ en découle. 
Pour trouver des nombres $a_{\gamma, \delta}$ vérifiant la relation (7), il suffit donc de résoudre le système linéaire $(7)$, d'inconnues $a_{\gamma, \delta}$ lorsque $\ell$ décrit $K(\mathcal{L})=A(\tau)_{20}$ (c'est le Lemme 2.7). Les $a_{\gamma, \delta}$ s'écrivent donc comme des valeurs en $\tau$ de quotients de formes modulaires de même poids, admettant des développements en série de Laurent à coefficients dans $\overline{\mathbb{Q}}$. Les arguments développés dans [Dav1, §4], permettent donc de conclure que les $a_{\gamma, \delta}$ sont bien des nombres algébriques, de hauteur $\leq c_{22} h$, éléments d'une extension de $k$ de degré sur $k \leq c_{22}$. Le Lemme 2.9 est donc entièrement démontré (bien entendu, le Lemme 2.9 peut être remplacé par le Lemme 2.6, mais outre le fait que sa preuve est plus simple, il permet d'écrire $f(\ell) / \theta_{\beta}\left(\tau, \frac{1}{2} \ell\right) \theta_{\beta_{N}}\left(\tau, N \frac{1}{2} \ell\right)$ comme une fraction rationnelle de degré un peu plus faible en les coordonnées des multiples de $P$, ce qui peut être utile).

Par induction, on peut donc conclure que $f(\ell)$ peut s'écrire comme une forme linéaire :

$$
\begin{aligned}
& \frac{f(\ell)}{\theta_{\beta}\left(\tau, \frac{1}{2} \ell\right) \theta_{\beta_{N}}\left(\tau, N \frac{1}{2} \ell\right)} \\
& \quad=\sum_{k=0}^{\kappa-1} 2^{k} \sum_{\gamma \in \mathcal{Z}_{2}, \delta \in \mathcal{Z}_{4}} a_{\gamma, \delta} \frac{\theta_{\gamma}\left(\tau, 2^{k} \frac{1}{2} \ell\right) \theta_{\delta}\left(\tau, 2^{k+1} \frac{1}{2} \ell\right)}{\theta_{\beta_{k}}\left(\tau, 2^{k} \frac{1}{2} \ell\right) \theta_{\delta_{k}}\left(\tau, 2^{k+1} \frac{1}{2} \ell\right)}
\end{aligned}
$$

(rappelons que $N$ est une puissance de 2 , soit $N=2^{\kappa}$; nous notons $\beta_{k}$ un élément de $\mathcal{Z}_{2}$ tel que $\left|\theta_{\beta_{k}}\left(\tau, 2^{k} \frac{1}{2} \ell\right)\right|$ soit maximal et $\delta_{k}$ un élément de $\mathcal{Z}_{4}$ tel que $\left|\theta_{\delta_{k}}\left(\tau, 2^{k+1} \frac{1}{2} \ell\right)\right|$ soit maximal, pour $\left.0 \leq k \leq \kappa\right)$. On en déduit donc que :

$$
\frac{f(\ell)}{\theta_{\beta}\left(\tau, \frac{1}{2} \ell\right) \theta_{\beta_{N}}\left(\tau, N \frac{1}{2} \ell\right)}
$$

est algébrique, élément d'une extension de $k$ de degré au plus $c_{44}$. Il reste à majorer sa hauteur : soient $X=\left(x_{0}, \ldots, x_{n}\right)$ et $Y=\left(y_{0}, \ldots, y_{n}\right)$, deux points de $\mathbb{P}^{n}(\overline{\mathbb{Q}})$ (normalisés de telle sorte que tous les $x_{i}$ (resp. tous les $y_{i}$ ) soient algébriques). On a alors l'inégalité triviale :

$$
h(X Y)=h\left(\sum_{i=0}^{n} x_{i} y_{i}\right) \leq h(X)+h(Y)+\log (n) .
$$

On en déduit, en posant :

$$
\begin{aligned}
& X=\left(\frac{\theta_{\gamma}\left(\tau, 2^{k} \frac{1}{2} \ell\right) \theta_{\delta}\left(\tau, 2^{k+1} \frac{1}{2} \ell\right)}{\theta_{\beta_{k}}\left(\tau, 2^{k} \frac{1}{2} \ell\right) \theta_{\delta_{k}}\left(\tau, 2^{k+1} \frac{1}{2} \ell\right)}\right)_{\gamma \in \mathcal{Z}_{2}, \delta \in \mathcal{Z}_{4}, 0 \leq k \leq \kappa-1}, \\
& Y=\left(2^{k} a_{\gamma, \delta}\right)_{\gamma \in \mathcal{Z}_{2}, \delta \in \mathcal{Z}_{4}, 0 \leq k \leq \kappa-1}, \\
& \text { TOME } 121-1993-\mathrm{N}^{\circ} 4
\end{aligned}
$$


que l'on a :

$$
h\left(\frac{f(\ell)}{\theta_{\beta}\left(\tau, \frac{1}{2} \ell\right) \theta_{\beta_{N}}\left(\tau, N \frac{1}{2} \ell\right)}\right)=h(X Y) \leq h(X)+h(Y)+\log \left(\kappa 2^{2 g} 2^{4 g}\right) .
$$

En remarquant que $Y$ est obtenu par un Segre à partir de

$$
Y_{0}=\left(1,2, \ldots, 2^{\kappa-1}\right) \quad \text { et } \quad Y_{1}=\left(a_{\gamma, \delta}\right)_{\gamma \in \mathcal{Z}_{2}, \delta \in \mathcal{Z}_{4}}
$$

on en déduit que $h(Y)=h\left(Y_{0}\right)+h\left(Y_{1}\right) \leq(\kappa-1) \log (2)+6 g \log (2) c_{22} h$ (en majorant la hauteur de $Y_{1}$ par la somme de celles de ses coordonnées, qui sont contrôlées par le LEMme 2.9 ). Il reste à estimer la hauteur de $X$. Notons $g: \mathbb{P}^{2^{2 g}-1} \times \mathbb{P}^{2^{4 g}-1} \rightarrow \mathbb{P}^{2^{6 g}-1}$ le Segre défini par :

$\nwarrow \mathrm{OK}$ ?

$$
\left(\left(a_{0}, \ldots, a_{2^{2 g}}\right),\left(b_{0}, \ldots, b_{2^{4 g}}\right)\right) \longmapsto\left(\ldots, a_{i} b_{j}, \ldots\right)_{0 \leq i \leq 2^{2 g}, 0 \leq j \leq 2^{4 g}}
$$

Notons pour tout entier $k$ compris entre 0 et $\kappa-1$,

- $A_{k}$ le point projectif $A_{k}=\left(\frac{\theta_{\gamma}\left(\tau, 2^{k} \frac{1}{2} \ell\right)}{\theta_{\beta_{k}}\left(\tau, 2^{k} \frac{1}{2} \ell\right)}\right)_{\gamma \in \mathcal{Z}_{2}}$,

- $B_{k}$ le point projectif $B_{k}=\left(\frac{\theta_{\delta}\left(\tau, 2^{k+1} \frac{1}{2} \ell\right)}{\theta_{\delta_{k}}\left(\tau, 2^{k+1} \frac{1}{2} \ell\right)}\right)_{\delta \in \mathcal{Z}_{4}}$.

Le point $X$ est alors obtenu à partir de $X_{0}=\left(A_{0}, \ldots, A_{\kappa-1}\right) \in\left(\mathbb{P}^{2^{2 g}-1}\right)^{\kappa}$ et de $X_{1}=\left(B_{0}, \ldots, B_{\kappa-1}\right) \in\left(\mathbb{P}^{2^{4 g}-1}\right)^{\kappa}$ par la flèche :

$$
\begin{aligned}
& \left(\mathbb{P}^{2^{2 g}-1}\right)^{\kappa} \times\left(\mathbb{P}^{2^{4 g}-1}\right)^{\kappa} \longrightarrow\left(\mathbb{P}^{2^{6 g}-1}\right)^{\kappa} \\
& \left(\left(A_{0}, \ldots, A_{\kappa-1}\right),\left(B_{0}, \ldots, B_{\kappa-1}\right)\right) \longmapsto\left(g\left(A_{0}, B_{0}\right), \ldots, g\left(A_{\kappa-1}, B_{\kappa-1}\right)\right) .
\end{aligned}
$$

Donc :

$$
h(X) \leq h\left(X_{0}\right)+h\left(X_{1}\right) \leq \sum_{i=0}^{\kappa-1} h\left(A_{i}\right)+h\left(B_{i}\right) .
$$

Le point $A_{0}$ est un point de 4 -division de $P$ et $A_{i}=2^{i} A_{0}$. De même, $B_{0}$ est un point de 4-division de $P$, mais dans le plongement de $A(\tau)$ dans $\mathbb{P}^{4^{2 g}-1}$ induit par $\mathcal{L}^{\otimes 16}$ (et fixé par la base de $\Gamma\left(A(\tau), \mathcal{L}^{\otimes 16}\right)$, donnée par les $\left.\left(\theta_{\delta}(\tau, 4 z)\right)_{\delta \in \mathcal{Z}_{4}}\right)$. En notant $h_{\mathcal{L} \otimes 16}=\max \left\{1, h\left(\left(\theta_{\delta}(\tau, 0)\right)_{\delta \in \mathcal{Z}_{4}}\right)\right\}$ 
et $\widehat{h}_{\mathcal{L} \otimes 16}$ la hauteur de Néron-Tate associée à ce nouveau plongement (trivialement, $\widehat{h}_{\mathcal{L}^{\otimes 16}}=4 \widehat{h}$ ), on obtient via le théorème $3-2$ de $[\mathrm{M}-\mathrm{Z}]$ :

$$
h(X) \leq\left(1+4+\cdots+4^{\kappa-1}\right)\left(\widehat{h}\left(A_{0}\right)+\widehat{h}_{\mathcal{L} \otimes 16}\left(B_{0}\right)\right)+c_{23} \kappa\left(h+h_{\mathcal{L} \otimes 16}\right) .
$$

Il reste à majorer $h_{\mathcal{L}^{\otimes 16}}$, ce qui se fait encore à l'aide des formules d'additions. Soit $\delta \in \mathcal{Z}_{4}$ :

$$
\theta_{\delta}(\tau, 0)^{4}=\frac{1}{2^{g}} \sum_{a \in \mathcal{Z}_{2}} \exp \left(-4 i \pi \delta_{1} a_{2}^{\prime}\right) \theta_{2 \delta+a}(\tau, 0) \theta_{a}(\tau, 0)^{3} .
$$

On en déduit donc aisément :

$$
h_{\mathcal{L} \otimes 16} \leq h+\frac{1}{2} g \log (2) .
$$

On obtient ainsi en combinant les inégalités précédentes

$$
h\left(\frac{f(\ell)}{\theta_{\beta}\left(\tau, \frac{1}{2} \ell\right) \theta_{\beta_{N}}\left(\tau, N \frac{1}{2} \ell\right)}\right) \leq c_{24}\left(N^{2} \widehat{h}(Q)+\log (N) h\right),
$$

ce qui achève la preuve de la Proposition 2.8 .

\section{Construction de transcendance}

Soient $A(\tau)$ une variété abélienne vérifiant les hypothèses du THÉORÈmE 1.4 et $P$ un point de $A(\tau)(k)$. Pour tout nombre réel $x$, nous noterons $[x]$ sa partie entière. Nous désignerons par $C_{0}$, une constante (i.e. un nombre réel positif ne dépendant que de $g$ ), plus grande que toutes les constantes $c_{1}, c_{2}, \ldots$ intervenant dans la démonstration. Fixons les paramètres suivants :

$$
\begin{array}{ll}
L=\left[C_{0}^{16 g} \rho \log (2 \rho)^{g+1}\right], & T=\left[C_{0}^{1 /(g+1)} L^{1+1 /(g+1)}\right], \\
R=C_{0}^{4} D(h+\log D) \log (2 \rho), & \mathcal{C}=C_{0}^{16 g}(\rho \log (2 \rho))^{g}, \\
U=T R / C_{0}^{3} &
\end{array}
$$

et soit $N$ la plus petite puissance de 2 supérieure ou égale à $C_{0} L$ (on a bien entendu $\left.N \leq 2 C_{0} L\right)$. Nous supposerons dans tout ce qui suit que :

$$
\widehat{h}(P) \leq C_{0}^{-66 g} \rho^{-(4 g+2)}(\log (2 \rho))^{-4 g-1} h .
$$

Rappelons que $\rho(A, k)=\rho=\frac{D(h+\log D)}{Y}+D^{1 /(g+2)}$.

$$
\text { TOME } 121-1993-\mathrm{N}^{\circ} 4
$$


Nous allons donc en déduire que $P$ est d'ordre fini modulo une sousvariété abélienne de $A$.

On peut supposer que le corps $k$ est la plus petite extension de $\mathbb{Q}$ sur laquelle $A$ et $P$ sont rationnels. Comme les coordonnées de l'origine $\left(\theta_{\alpha}(\tau, 0)\right)_{\alpha \in \mathcal{Z}_{2}}$ engendrent le corps de définition de $A(\tau)$ (voir par exemple [Ma1, p. 114]),

$$
k=\mathbb{Q}\left[\left(\theta_{\alpha}(\tau, 0)\right)_{\alpha \in \mathcal{Z}_{2}},\left(\theta_{\beta}(\tau, 2 s)\right)_{\beta \in \mathcal{Z}_{2}}\right]
$$

(où $s$ est un élément de $\mathbb{C}^{g}$ tel que $\Theta_{\tau}(s)=P$ ), il existe alors une base $\mathcal{E}$ de $k$ sur $\mathbb{Q}$ formée de monômes de degré au plus $D$ en les nombres

$$
\frac{\theta_{\beta}(\tau, 0)}{\theta_{\alpha}(\tau, 0)}, \quad \frac{\theta_{\gamma}(\tau, 2 s)}{\theta_{\delta}(\tau, 2 s)},
$$

pour $\beta$ et $\gamma$ décrivant $\mathcal{Z}_{2}$ ( $\delta$ étant un élément de $\mathcal{Z}_{2}$ tel que $\left|\theta_{\delta}(\tau, 2 s)\right|$ soit maximal). Nous noterons $b_{i}$, pour $1 \leq i \leq[k: \mathbb{Q}]$, les éléments de cette base. La hauteur des éléments $\left(b_{i}\right)_{1 \leq i \leq[k: \mathbb{Q}]}$ de $\mathcal{E}$ est alors majorée par $c_{25} D(h(P)+h(A(\tau))$, où $h(P)$ est la hauteur de Weil de $P$. Par le théorème 3 -2 de [M-Z], on a alors :

$$
\forall i, 1 \leq i \leq D, \quad h\left(b_{i}\right) \leq c_{26} D\{h(A(\tau))+\widehat{h}(P)\} \leq c_{27} D h .
$$

\subsection{Construction de la fonction auxiliaire.}

Fixons $s=s^{(1)} \tau+s^{(2)}$, où $s^{(1)}$ et $s^{(2)}$ sont des vecteurs de $\mathbb{R}^{g}$ de coordonnées toutes comprises entre 0 et 1 , tels que $\Theta_{\tau}(s)=P$. Le principe des tiroirs permet d'affirmer qu'il y a au moins un multiple $m s$ de $s$ (avec $-\mathcal{C} \leq m \leq \mathcal{C}$ ) tel que la partie fractionnaire de la dernière coordonnée de $m s^{(1)}$ soit $\leq 1 / \mathcal{C}$. Nous noterons $\ell=\ell^{(1)} \tau+\ell^{(2)}$ le représentant de $m s^{(1)}$ (pour fixer les idées, on choisit pour $m$ le plus petit entier vérifiant la propriété ci-dessus) modulo le réseau des périodes $\Lambda$ dont toutes les coordonnées de $\ell_{1}$ et de $\ell_{2}$ sont réelles, comprises entre 0 et 1 , et nous poserons $Q=\Theta_{\tau}(\ell)=Q$. Le point $Q$ de $A(\tau)$ est donc un multiple de $P$ et $\widehat{h}(Q) \leq \mathcal{C}^{2} \widehat{h}(P)$.

Soit maintenant $\mathbb{G}$,

$$
0 \rightarrow \mathbb{G}_{m}^{2} \longrightarrow \mathbb{G} \longrightarrow A(\tau) \rightarrow 0
$$

l'extension de $A(\tau)$ par $\mathbb{G}_{m}^{2}$ paramétrée par $(\ell, N \ell)$ (i.e. le point $(Q, N Q)$ de $A(\tau)^{2}$ identifiée à sa duale). On notera que si l'on a imposé aux coordonnées de $\ell$ d'être comprises entre 0 et 1 , le deuxième vecteur paramétrant notre groupe n'a pas été normalisé. L'espace tangent à 
l'origine de $\mathbb{G}$ étant identifié à $\mathbb{C}^{g+2}$, nous disposons d'une base naturelle de l'espace tangent à l'origine de $\mathbb{G}$, notée $\left(\partial / \partial z_{1}, \ldots \partial / \partial z_{g}, \partial / \partial t_{1}, \partial / \partial t_{2}\right)$. Soit $\Delta$ le sous-espace de $T_{\mathbb{G}}(\mathbb{C})$ engendré par la base :

$$
u_{0}=\frac{\partial}{\partial t_{1}}+N \frac{\partial}{\partial t_{2}}, \quad u_{i}=\frac{\partial}{\partial z_{i}}-2 i \pi \ell_{i}^{(1)} u_{0}, \quad 1 \leq i \leq g
$$

$\left(\ell_{i}^{(1)}\right.$ est la $i$-ième coordonnée du vecteur $\left.\ell^{(1)}\right)$. On notera que les vecteurs $u_{i}$, pour $1 \leq i \leq g$, correspondent à des relevés dans $T_{\mathbb{G}}$ de périodes de $A(\tau)$; en effet, tout vecteur de $\mathbb{Z}^{g} \subset \Lambda$, se relève en une combinaison linéaire des $u_{i}$ dans $\Lambda^{\prime}$.

L'espace $\Delta$ correspond à l'espace tangent à l'origine du sous-groupe $\mathbb{G}^{\prime}$ de $\mathbb{G}$ défini par le diagramme :

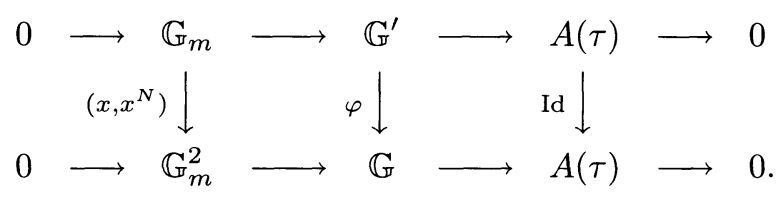

Choisissons maintenant un système $\underline{f}=\left(f_{0}, \ldots, f_{g+2}\right)$ homogènement algébriquement indépendant de fonctions (parmi les fonctions définies en (4)) de $\mathbb{C}^{g+2}$ dans $\mathbb{C}$ donnant le plongement projectif de $\mathbb{G}$.

Rappelons le lemme de Thue-Siegel qui va nous permettre de construire notre fonction auxiliaire :

LEMmE 3.1. - Soit $\left(u_{i, j}\right)_{1 \leq i \leq \nu, 1 \leq j \leq \mu}$ une matrice de nombres complexes, de rang $\leq \eta$. Soient $\delta, m, p$ des nombres réels positifs tels que:

$$
\begin{gathered}
{\left[2 \mu \mathrm{e}^{\delta+m+p}+1\right]^{2 \eta} \leq \mathrm{e}^{\nu \delta}} \\
\max \left\{\sum_{j=1}^{\nu}\left|u_{i, j}\right|, 1 \leq j \leq \nu\right\} \leq \mathrm{e}^{m} .
\end{gathered}
$$

Alors, il existe $\left(a_{1}, \ldots, a_{\nu}\right) \in \mathbb{Z}^{\nu}$ tel que:

$$
\begin{gathered}
0<\max \left\{\left|a_{i}\right|, 1 \leq i \leq \nu\right\} \leq \mathrm{e}^{\delta} \\
\max \left\{\left|\sum_{i=1}^{\nu} u_{i, j} a_{i}\right|, 1 \leq j \leq \mu\right\} \leq \mathrm{e}^{-p} .
\end{gathered}
$$

Voir [P-W, Lemme 6-1].

TOME $121-1993-\mathrm{N}^{\circ} 4$ 
Posons :

$$
\begin{array}{ll}
\mu=\eta=(T+1)^{g}\left(\left[T / C_{0}^{3}\right]+1\right), & \nu=D(L+1)^{g+2}, \\
m=p=U, & \delta=\frac{U}{C_{0} D} .
\end{array}
$$

Nous allons construire une fonction $F$

$$
F(\underline{z}, t)=P(\underline{f}(\underline{z}, t, N t)) ;
$$

où $P$ est un polynôme homogène de degré $\leq L$, à coefficients de la forme $\sum_{i=1}^{i=D} \alpha_{i} b_{i}$, où les $\alpha_{i}$ sont des entiers et les $b_{i}$ sont des éléments de $\mathcal{E}$, vérifiant la propriété

$$
\left|\Delta_{u} F(0)\right| \leq \exp (-U)
$$

pour toute dérivation $\Delta_{u}=D_{u_{0}}^{t_{0}} \circ \cdots \circ D_{u_{g}}^{t_{g}}$, où les entiers $t_{i}$ vérifient les inégalités $0 \leq t_{i} \leq T$ pour $0 \leq i \leq g-1$ et $0 \leq t_{g} \leq T / C_{0}^{3}$. Il s'agit donc de résoudre un système d'inéquations linéaires de la forme (11). Pour assurer l'existence d'une solution, il suffit de vérifier les conditions (8) et (9) du Lemme 3.1. Avec les paramètres que nous avons fixés, la condition (8) se déduit de

$$
\frac{T^{g+1}}{C_{0}^{3}} \leq \frac{c_{28}}{C_{0}^{2}} L^{g+2}
$$

qui est clairement satisfaite. Pour vérifier la condition (9), il suffit de montrer que :

$$
\begin{aligned}
& D(L+1)^{g+2} \max \left\{\left|b_{i}\right|, 1 \leq i \leq D\right\} \\
& \times \max \left\{\left|\Delta_{u}\left(f_{0}^{L_{0}} \cdots f_{g+2}^{L_{g}+2}(0)\right)\right|, \Delta_{u}, \sum_{i=0}^{g+2} L_{i} \leq L\right\} \\
& \leq \exp (U) .
\end{aligned}
$$

Pour ceci, notons $A$ la norme du sup de la matrice donnant les coordonnées des vecteurs $u_{i}$ (on a $A \leq c_{29} N$ ), et posons $f=f_{0}^{L_{0}} \cdots f_{g+2}^{L_{g+2}}$ pour alléger l'écriture. On a alors :

$$
\begin{array}{r}
\max \left\{\left|\Delta_{u}(f(0))\right|, f, \Delta_{u}\right\} \\
\leq\left(c_{30} A\right)^{(g+1) T} \max \left\{\left|\frac{\partial t_{1}}{\partial z_{1}} \circ \cdots \circ \frac{\partial t_{g}}{\partial z_{g}} \circ \frac{\partial t_{g+1}}{\partial t_{1}} \circ \frac{\partial t_{g+2}}{\partial t_{2}}(f(0))\right| f,\right. \\
\left.\quad 0 \leq t_{i} \leq(g+1) T, 1 \leq i \leq g+2\right\}
\end{array}
$$

(voir par exemple [P-W, lemme 3-1]). 
Cette inégalité, jointe aux inégalités de Cauchy, donne alors :

$$
\begin{aligned}
\log \left|\Delta_{u}(f(0))\right| \leq c_{31}( & T \log T+T \log N \\
& +L \log \left(\max \left\{\left|f_{i}\left(z, t_{1}, t_{2}\right)\right|,\left|\left(z, t_{1}, t_{2}\right)\right|=1\right\}\right) .
\end{aligned}
$$

Ici, $\left|\left(z, t_{1}, t_{2}\right)\right|$ est la norme «naturelle» sur $\mathbb{C}^{g+2}$, i.e.

$$
\left|\left(z, t_{1}, t_{2}\right)\right|=\left\{\sum_{i=1}^{g}\left|z_{i}\right|^{2}+\left|t_{1}\right|^{2}+\left|t_{2}\right|^{2}\right\}^{1 / 2} .
$$

Par ailleurs, le lemme de croissance 2.4 nous assure que :

$$
\left|f_{i}\left(z, t_{1}, t_{2}\right)\right| \leq \exp \left(c_{33} Y\right) \quad \forall i, 0 \leq i \leq g+2,\left|\left(z, t_{1}, t_{2}\right)\right| \leq 1
$$

(rappelons que $\left\|\operatorname{Im} \tau^{-1}\right\| \leq c_{32}$, voir [Ig, Lemme 15, p. 195]). Enfin, l'inégalité de la taille nous assure que :

$$
\max \left\{\left|b_{i}\right|, 1 \leq i \leq D\right\} \leq \exp \left(c_{26} D^{2} h\right)
$$

En combinant ces inégalités, on peut en déduire que :

$$
\log \mid \Delta_{u}\left(f(0) \mid \leq c_{31}(T \log T+T \log N+L Y)\right.
$$

Le choix des paramètres, ainsi que le lemme matriciel de D. Masser, (voir [Ma1, lemme matriciel]) permettent d'assurer que :

$$
\log L+\log D+D^{2} h+T \log T+T \log N+L Y \leq \frac{U}{\sqrt{C}_{0}} .
$$

L'inégalité (14) est donc exacte, ce qui assure la validité de la condition (9).

Le LEMME 3.1 nous permet alors d'affirmer qu'il existe des nombres entiers $\alpha_{i}$ non tous nuls tels que $\left|\alpha_{i}\right| \leq \exp \left(U /\left(C_{0} D\right)\right)$, tels que la fonction auxiliaire $F$ vérifie les conditions (12).

3.2. Extrapolation. - Nous allons utiliser la méthode de Gel'fond pour extrapoler sur l'ordre d'annulation de la fonction $F$ en $(z, t)=0$. Pour cela, nous utiliserons le Lemme 2.2, qui permet d'affirmer que la croissance des fonctions quasi-abélienne est «ralentie» sur la direction $u_{g}$.

Soit $g$ la fonction d'une variable, définie par

$$
g(z)=\Delta_{u}\left(F\left(z u_{g}\right)\right)
$$

TOME $121-1993-\mathrm{N}^{\circ} 4$ 
où $\Delta_{u}$ est cette fois de la forme

$$
\Delta_{u}=D_{u_{0}}^{t_{0}} \circ \cdots \circ D_{u_{g-1}}^{t_{g-1}}
$$

avec $0 \leq t_{i}, 0 \leq i \leq g-1$ et $\sum_{i=0}^{g-1} t_{i} \leq T$. Par périodicité (les facteurs d'automorphie sont égaux à 1 ) de $F$ par rapport à $\mathbb{Z} u_{g}$, on en déduit que la fonction $g(z)$ vérifie

$$
\left|\frac{\partial^{k}}{\partial z^{k}} g(n)\right| \leq \exp (-U)
$$

pour tout $k$ tel que $0 \leq k \leq T / C_{0}^{3}$ et tout entier $n$ de $\mathbb{Z}$. Nous pouvons donc appliquer la formule d'interpolation classique suivante :

LEMME 3.2. - Soit $g$ une fonction analytique dans le disque $|z| \leq R d u$ plan complexe; soient $2 \leq r \leq \frac{1}{2} R$ et $T_{1}, S_{1}$ deux entiers positifs. Alors:

$$
\begin{aligned}
|g|_{2 r} \leq 2|g|_{R}\left(\frac{4 r}{R}\right)^{T_{1} S_{1}} & \\
& +5\left(\frac{18 r}{S_{1}}\right)^{T_{1} S_{1}} \max \left\{\left|\frac{g^{(t)}(s)}{t !}\right| ; 0 \leq t \leq T_{1}, 0 \leq s \leq S_{1}\right\} .
\end{aligned}
$$

Nous avons utilisé ici les notations usuelles:

$$
|g|_{R}=\sup _{|z|=R}\{|g(z)|\} \quad \text { et } \quad g^{(t)}=\frac{\mathrm{d}^{t} g}{\mathrm{~d} z^{t}} \quad(t \in \mathbb{N}) .
$$

Voir [W, lemme 2-3].

Appliquons ce lemme avec $T_{1}=\left[T / C_{0}^{3}\right], r=\frac{1}{8} R$ et $S_{1}=[r]$. Il reste à estimer $|g|_{R}$, ce qui peut se faire à partir des inégalités de Cauchy; toutefois, pour ne pas perdre les propriétés de «ralentissement» de la croissance des fonctions quasi abélienne le long de la direction $u_{g}$, il faut appliquer ces inégalités au «voisinage» de la droite $\mathbb{C} u_{g}$ et non pas sur un polydisque de rayon $R$ (voir aussi [Ma-Wü, lemme 4-3]) : soient $\varepsilon$ un nombre réel strictement positif et $z$ un nombre complexe. On a alors :

$$
\begin{aligned}
\left|g\left(z u_{g}\right)\right| & =\left|\Delta_{u}\left(F\left(z u_{g}\right)\right)\right| \\
& \leq \frac{T !}{\varepsilon^{T}} \max \left\{\left|F\left(\varepsilon_{1} u_{1}, \varepsilon_{2} u_{2}, \ldots, \varepsilon_{g-1} u_{g-1}, z u_{g}\right)\right|\right. \\
& \left.\left|\varepsilon_{i}\right|=\varepsilon, 1 \leq i \leq g-1\right\}
\end{aligned}
$$

BULLETIN DE LA SOCIÉTÉ MATHÉMATIQUE DE FRANCE 
Supposons $|z| \leq R$. Le lemme de croissance 2.4 donne alors :

$$
\begin{aligned}
& \log |g|_{R} \leq c_{34}\{- \log (\varepsilon)+T \log T+\frac{U}{C_{0} D}+D^{2} h+L R^{2} H\left(e_{g}, e_{g}\right) \\
&+L \varepsilon^{2} \sum_{\substack{1 \leq i \leq g-1 \\
1 \leq j \leq g-1}} H\left(e_{i}, e_{j}\right)+L R \varepsilon \sum_{1 \leq i \leq g-1} H\left(e_{i}, e_{g}\right) \\
&\left.+L Y+\frac{L R}{\mathcal{C}}+\frac{L N R}{\mathcal{C}}+L \varepsilon+L N \varepsilon\right\}
\end{aligned}
$$

Posons maintenant $\epsilon=1 / T$. Le Lemme 2.2 donne $H\left(e_{g}, e_{g}\right) \leq c_{12} / Y$, et on a $H\left(e_{i}, e_{j}\right) \leq c_{32}$ puisque $\left\|\operatorname{Im} \tau^{-1}\right\| \leq c_{32}$; d'où :

$$
\log |g|_{R} \leq c_{35}\left(T \log T+\frac{L R^{2}}{Y}+D^{2} h+\frac{U}{C_{0} D}+L Y+\frac{L N R}{\mathcal{C}}\right)
$$

C'est-à-dire, comme $L R^{2} / Y \leq\left(c_{36} / C_{0}\right) U, L N R / \mathcal{C} \leq\left(c_{37} / C_{0}\right) U$, (de même que les autres termes qui interviennent dans le membre de droite de l'inégalité ci-dessus),

$$
|g|_{R} \leq \exp \left(\frac{c_{38} U}{C_{0}}\right)
$$

Comme $T_{1} S_{1} \geq c_{39} U$ et $T_{1} S_{1} \log \left(18 r / S_{1}\right) \leq \frac{1}{2} U$, la formule d'interpolation nous donne:

$$
|g|_{2 r} \leq \exp \left(-c_{40} U\right)+5\left(\frac{18 r}{S_{1}}\right)^{T_{1} S_{1}} \exp (-U) \leq \exp \left(-c_{41} U\right) .
$$

Les inégalités de Cauchy permettent alors d'écrire :

$$
\left|g^{(t)}(0)\right| \leq \frac{t !}{R^{t}}|g|_{R}, \quad \forall t, 0 \leq t, \forall R, 0<R
$$

Le choix des paramètres donne alors :

$$
\left|g^{(t)}(0)\right| \leq \exp \left(-c_{42} U\right), \quad \forall t, 0 \leq t \leq T .
$$

3.3. Passage à une base algébrique. - Nous avons donc établi que

$$
\left|\Delta_{u}(F(0))\right| \leq \exp \left(-c_{42} U\right), \quad \Delta_{u}=D_{u_{0}}^{t_{0}} \circ \cdots \circ D_{u_{g}}^{t_{g}}
$$

pour $0 \leq t_{i}, 0 \leq i \leq g$ tels que $\sum_{i=0}^{g} t_{i} \leq T$.

TOME $121-1993-\mathrm{N}^{\circ} 4$ 
Nous allons maintenant repasser dans une base algébrique de l'espace de dérivations. Pour $1 \leq i \leq g$ et $d_{0}=u_{0}$, posons :

$$
d_{i}=\delta_{i}-\left(2 i \pi A_{i} \ell^{(1)^{\prime}}+4 \frac{\delta_{i} \theta_{\beta}\left(\tau, \frac{1}{2} \ell\right)}{\theta_{\beta}\left(\tau, \frac{1}{2} \ell\right)}\right)\left(\frac{\partial}{\partial t_{1}}+N \frac{\partial}{\partial t_{2}}\right)
$$

La matrice de passage $M$ entre la base de dérivations donnée par les $\left(u_{i}\right)_{0 \leq i \leq g}$ et la base de dérivations $\left\{d_{i}\right\}$ vaut :

$$
M=\left(\begin{array}{c|ccc}
1 & 0 & \cdots & 0 \\
-4 \frac{\delta_{1} \theta_{\beta}\left(\tau, \frac{1}{2} \ell\right)}{\theta_{\beta}\left(\tau, \frac{1}{2} \ell\right)} & & & \\
\vdots & & P(\tau) \\
-4 \frac{\delta_{g} \theta_{\beta}\left(\tau, \frac{1}{2} \ell\right)}{\theta_{\beta}\left(\tau, \frac{1}{2} \ell\right)} & &
\end{array}\right)
$$

La norme de $M$ est donc $\leq \exp \left(c_{43} D h\right.$ ) (pour une majoration de la norme de $P(\tau)$, on pourra voir [Dav1, lemme 4-14]; les autres coefficients de la matrice sont faciles à majorer en utilisant le fait que les coordonnées du vecteur $\ell^{(1)}$ sont majorés par un, et en utilisant le lemme de croissance 2.1). On en déduit (cf. [P-W, lemme 3-1]) que :

$$
\left|\Delta^{T}(F(0))\right| \leq \exp \left(c_{43} T D h-c_{42} U\right) \leq \exp \left(-c_{44} U\right),
$$

avec $\Delta^{T}=d_{0}^{t_{0}} \circ \cdots \circ d_{g}^{t_{g}}$ et $\sum t_{i} \leq T$. De plus, on a la relation :

$$
\begin{gathered}
d_{i}-\delta_{i}^{\prime}=\delta_{i}-\left(2 i \pi A_{i} \ell^{(1)^{\prime}}+4 \frac{\delta_{i}\left(\theta_{\beta}\left(\tau, \frac{1}{2} \ell\right)\right)}{\theta_{\beta}\left(\tau, \frac{1}{2} \ell\right)}\right)\left(\frac{\partial}{\partial t_{1}}+N \frac{\partial}{\partial t_{2}}\right) \\
-\left\{\delta_{i}-\left(2 i \pi A_{i} \ell^{(1)^{\prime}}+4 \frac{\delta_{i}\left(\theta_{\beta}\left(\tau, \frac{1}{2} \ell\right)\right)}{\theta_{\beta}\left(\tau, \frac{1}{2} \ell\right)}\right) \frac{\partial}{\partial t_{1}}\right. \\
\left.-\left(2 i N \pi A_{i} \ell^{(1)^{\prime}}+4 \frac{\delta_{i}\left(\theta_{\beta_{N}}\left(\tau, N \frac{1}{2} \ell\right)\right)}{\theta_{\beta_{N}}\left(\tau, N \frac{1}{2} \ell\right)}\right) \frac{\partial}{\partial t_{2}}\right\} \\
=4\left(-N \frac{\delta_{i}\left(\theta_{\beta}\left(\tau, \frac{1}{2} \ell\right)\right)}{\theta_{\beta}\left(\tau, \frac{1}{2} \ell\right)}+\frac{\delta_{i}\left(\theta_{\beta_{N}}\left(\tau, N \frac{1}{2} \ell\right)\right)}{\theta_{\beta_{N}}\left(\tau, N \frac{1}{2} \ell\right)}\right) \frac{\partial}{\partial t_{2}} .
\end{gathered}
$$

La base $\left\{d_{i}, 0 \leq i \leq g\right\}$ est donc algébrique en vertu de la ProposiTION 2.8. 
Soient maintenant :

- $g$ le quotient $g(z)=F / \theta_{\alpha}(\tau, 2 z)^{m}$, où $\alpha$ est choisi dans $\mathcal{Z}_{2}$ de telle sorte que $\left|\theta_{\alpha}(\tau, 0)\right|$ soit maximal;

- $\Delta$ un monôme différentiel $d_{0}^{t_{0}} \circ \cdots \circ d_{g}^{t_{g}}$ (avec $\sum t_{i} \leq T$ ) minimal pour lequel $\Delta(F(0))$ est non nul;

- $m$ le degré de $P$.

La formule de Leibnitz nous donne :

$$
\Delta(g(0))=\frac{\Delta(F(0))}{\theta_{\alpha}(\tau, 0)^{m}}
$$

et le Lemme 2.1 nous assure que :

$$
|\Delta(g(0))| \leq \exp \left(-c_{45} U\right)
$$

Comme le membre de gauche est algébrique, l'inégalité de la taille nous montre qu'en fait ce dernier est nul : une récurrence facile à partir du Lemme 2.8 et de la Proposition 2.5 (et du théorème 4-2 de [Dav1] pour le calcul de $\left.\theta_{m}(\tau, 2 z) \delta_{i}\left(\theta_{n}(\tau, 2 z)\right)-\theta_{n}(\tau, 2 z) \delta_{i}\left(\theta_{m}(\tau, 2 z)\right)\right)$ permet alors de conclure que la hauteur de $\Delta(g(0))$ est :

$$
\begin{aligned}
& \leq c_{46}\left\{(T+L) \log (T+L)+T\left(\log (N) h+N^{2} \widehat{h}(Q)\right)+L h+\frac{U}{C_{0} D}\right\} \\
& \leq c_{47} T\left(\log (N) h+N^{2} \mathcal{C}^{2} \widehat{h}(P)\right) \\
& \leq c_{48} T \log (\rho) h \\
& \leq c_{49} U .
\end{aligned}
$$

Le choix des paramètres, l'inégalité de la taille ainsi que l'hypothèse faite sur $\widehat{h}(P)$ permettent donc de conclure que $\Delta^{T}(F(0))=0$ pour toute dérivation $\Delta$ d'ordre $\leq T$. En résumé, la fonction $F$ s'annule à l'origine, à l'ordre $T$ le long de la direction $\Delta$.

3.4. Lemme de zéros et conclusion. - Rappelons tout d'abord le théorème suivant, dû à P. PHILIPPON :

THÉORÈme 3.3. - Soit $\mathbb{G}$ un groupe algébrique commutatif défini sur $\mathbb{C}$, de dimension $n$, plongé de façon projectivement normale dans un espace projectif $\mathbb{P}^{N}$. On notera deg l'application degré sur les sous ensembles ouverts des fermés de $\mathbb{P}^{N}$. Soient encore $\Delta$ un sous-espace de $T_{\mathbb{G}}(\mathbb{C})$, l'espace tangent à l'origine de $\mathbb{G}$, et $S$ un ensemble fini de points de $\mathbb{G}$, contenant l'origine 0 . On note $\Gamma^{(n)}(S)$ l'ensemble $\left\{\sum_{i=1}^{n} P_{i}, P_{i} \in S\right\}$. Soit par ailleurs, un élément $P$ de $\mathbb{C}\left[X_{0}, \cdots, X_{N}\right]$, homogène de degré $L$, 
qui s'annule à l'ordre $n T+1$ le long de $\Delta$ sur $\Gamma^{(n)}(S)$, mais qui n'est pas identiquement nul sur $\mathbb{G}$. Il existe alors un sous-groupe algébrique connexe $B$ de $\mathbb{G}$, distinct de $\mathbb{G}$, tel que :

$$
\begin{aligned}
&\left(\begin{array}{c}
T+\operatorname{codim}_{\Delta}\left(\Delta \cap T_{B}\right) \\
\operatorname{codim}_{\Delta}\left(\Delta \cap T_{B}\right)
\end{array}\right) \operatorname{card}((S+B) / B) \operatorname{deg}(B) D^{\operatorname{dim}(B)} \\
& \leq \operatorname{deg}(\mathbb{G})(2 L)^{\operatorname{dim}(\mathbb{G})}
\end{aligned}
$$

Démonstration. - Voir [Ph, th. 2-1] joint à [La1]. Notons également que L. Denis [De] a remarqué récemment que cet énoncé restait valable (avec la constante 1) sans hypothèse sur le plongement, tout au moins lorsque $\mathbb{G}$ est une variété abélienne. Il est vraisemblable que sa preuve s'applique qu'au cas général.

Le lemme de zéros de Philippon permet maintenant de conclure : puisque $F$ s'annule le long de $\Delta$ à un ordre $\geq T$, il assure l'existence d'un sous-groupe algébrique $B$ connexe, propre de $\mathbb{G}$ tel que :

$$
T^{\operatorname{codim}_{\Delta}\left(T_{B} \cap \Delta\right)} \operatorname{deg}(B) \leq c_{50} L^{\operatorname{codim}(B)} .
$$

Nous allons faire l'analyse des sous-groupes possibles :

- $B=\{0\}$. Dans ce cas, on est conduit à l'inégalité $T^{g+1} \leq c_{50} L^{g+2}$, qui est fausse.

- $B$ est isomorphe à $\mathbb{G}_{m}$ et $T_{B} \cap \Delta=\{0\}$. Dans ce cas, on a l'inégalité $T^{g+1} \leq c_{50} L^{g+1}$, qui est également fausse.

- $B$ est isomorphe à $\mathbb{G}_{m}$ et $T_{B} \subset \Delta$. Dans ce cas, $B$ est le sousgroupe défini par l'équation $x_{2}=x_{1}^{N}$ et son degré est $N$ (voir par exemple [Be-Ph]). L'inégalité s'écrit alors $T^{g} N \leq c_{50} L^{g+1}$, et comme $N \geq C_{0} L$, cette inégalité est également fausse.

- $B$ est isomorphe à $\mathbb{G}_{m}^{2}$. Dans ce cas, on a $T^{g} \leq c_{50} L^{g}$, qui est également fausse.

- $B$ se projette surjectivement sur $A$, et la partie linéaire de $T_{B}$ (i.e. $\left.T_{B} \cap T_{\mathbb{G}_{m}^{2}}\right)$ n'est pas nulle. Dans ce cas, $B$ est isogène à une extension de $A$ par $\mathbb{G}_{m}$ ( $B$ est une sous-variété propre de $\mathbb{G}$ ). Distinguons deux sous-cas :

$\triangleright$ La partie linéaire de $T_{B}$ ne rencontre pas $\Delta$, l'inégalité s'écrit alors $T \leq c_{50} L$, qui est fausse.

$\triangleright$ La partie linéaire de $T_{B}$ rencontre $\Delta$. Dans ce cas, le sous-groupe $B$ est une extension du groupe $\mathbb{G}^{\prime}$ le long duquel nous avons travaillé par un groupe fini. Son degré est alors $\geq N \operatorname{deg}(A)$ (voir [La2]). On a alors $N \leq c_{50} L$, qui est fausse car $N \geq C_{0} L$. 
- $B$ est isogène à $A$. Nous noterons $i$ l'inclusion de $B$ dans $\mathbb{G}$ et $\varphi$ l'isogénie donnée par la composition $B \stackrel{i}{\longrightarrow} \mathbb{G} \rightarrow A$. Considérons alors le diagramme :

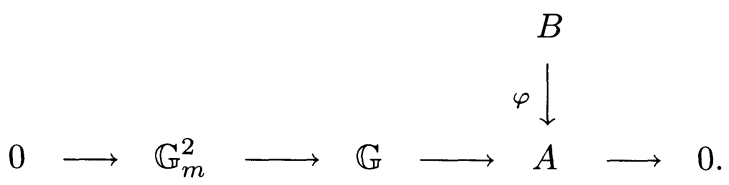

Soit $\widetilde{\mathbb{G}}$ l'image réciproque de $\mathbb{G}$ par $\varphi$, i.e. $\widetilde{\mathbb{G}}=\mathbb{G} \times_{A} B$. On a alors le diagramme :

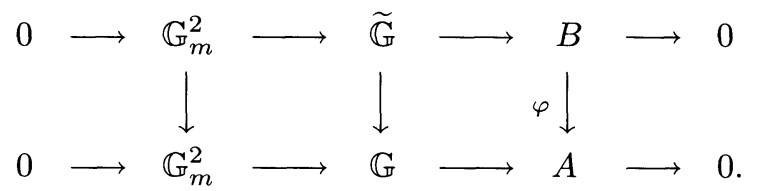

$\widetilde{\mathbb{G}}$ est donc l'extension de $B$ par $\mathbb{G}_{m}^{2}$ paramétrée par $\left(\varphi^{\vee}(Q), \varphi^{\vee}(N Q)\right)$ (voir par exemple $[\mathrm{Be}, \S 2]$ ), où $\varphi^{\vee}$ est l'isogénie duale

$$
A^{\vee} \stackrel{\varphi^{\vee}}{\longrightarrow} B^{\vee}
$$

induite par $\varphi$ (n'oublions pas que $A$ a été identifiée à sa duale $A^{\vee}$, via la polarisation principale). Par ailleurs, on a le diagramme :

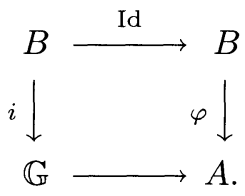

Par définition du produit fibré, on en déduit donc une flèche $s: B \longrightarrow \widetilde{\mathbb{G}}$ rendant commutatif le diagramme :

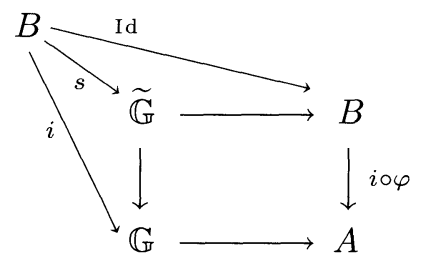

TOME $121-1993-\mathrm{N}^{\circ} 4$ 
et relevant donc la projection $\widetilde{\mathbb{G}} \rightarrow B$. L'extension $\widetilde{\mathbb{G}}$ est donc scindée, c'est-à-dire :

$$
\left(\varphi^{\vee}(Q), \varphi^{\vee}(N Q)\right)=(0,0) .
$$

Dans ce cas, $Q$ est un point d'ordre fini (soit $S$ l'ordre de $Q$ ) de $A$ et

$$
\begin{aligned}
\operatorname{deg}(B) & =c_{51} \operatorname{deg} \varphi & & (\text { voir [La2] }) \\
& =c_{51} \operatorname{deg} \varphi^{\vee} & & (\text { voir [Mum, p. 81] }) \\
& \geq c_{51} S & &
\end{aligned}
$$

et on obtient $T S \leq c_{52} L^{2}$, c'est-à-dire $S \leq c_{53} C_{0}^{16 g} \rho^{g}$. Le point $P$ est alors d'ordre $\leq c_{54} C_{0}^{32 g} \rho^{2 g}$. Le THÉORÈme 1.4 est donc exact dans ce cas.

- On peut donc maintenant supposer que $B$ se projette sur une sousvariété abélienne propre $B^{\prime}$ de $A$ (i.e. $0 \neq B^{\prime} \neq A$ ). Nous noterons $r$ la codimension de $B^{\prime}\left(r=\operatorname{codim}_{A}\left(B^{\prime}\right)\right)$. On peut comme précédement distinguer les sous-cas :

$\triangleright$ La partie linéaire de $T_{B}$ est de dimension 2. On a alors l'inégalité $T^{r} \leq c_{50} L^{r}$, qui est fausse.

$\triangleright$ La partie linéaire de $T_{B}$ est de dimension 1 , et ne rencontre pas $\Delta$. La relation $T^{r+1} c_{50} L^{r+1}$, est également fausse.

$\triangleright$ La partie linéaire de $T_{B}$ est de dimension 1 , et rencontre $\Delta$. La relation $T^{r} N \leq c_{50} L^{r+1}$, est aussi fausse.

$\triangleright$ La partie linéaire de $B$ est de dimension 0 . Dans ce cas, $B$ est isogène à une sous-variété abélienne $B^{\prime}$ de $A$ La restriction à $B$ de la projection $\mathbb{G} \rightarrow A$ induit une telle isogénie, que nous noterons $\varphi$. Nous noterons également $i^{\prime}$ l'inclusion $B^{\prime} \rightarrow A$ et $i$ l'inclusion $i: B \rightarrow \mathbb{G}$. Considérons alors comme précédemment le diagramme

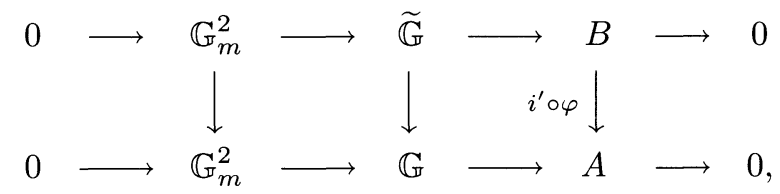

$\operatorname{avec} \widetilde{\mathbb{G}}=B \times_{A} \mathbb{G}$. Le diagramme commutatif

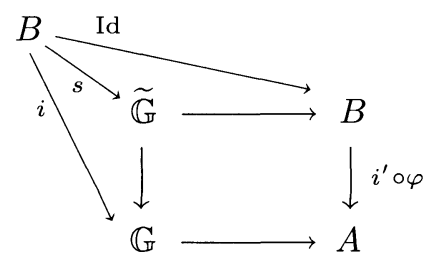


permet de voir comme précédemment que $\widetilde{\mathbb{G}}$ est scindée. Comme $\widetilde{\mathbb{G}}$ est paramétrée par $\left(\left(i^{\prime} \circ \varphi\right)^{\vee}(Q),\left(i^{\prime} \circ \varphi\right)^{\vee}(N Q)\right)$, on en déduit :

$$
\varphi^{\vee} \circ i^{\prime \vee}(Q)=0 \text {. }
$$

En d'autres termes, le point $Q$ est d'ordre fini $S$ modulo $\operatorname{ker}\left(i^{\prime \vee}\right)$ et

$$
S \leq \operatorname{deg}\left(\varphi^{\vee}\right)=\operatorname{deg}(\varphi) .
$$

La suite exacte

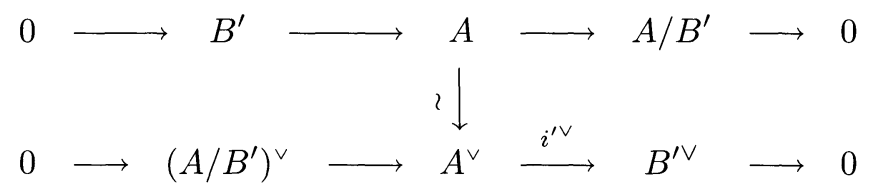

permet d'identifier $\left(A / B^{\prime}\right)^{\vee}$ à une sous-variété abélienne $B^{\prime \perp}$ de $A$ (on remarquera qu'analytiquement, si $A \simeq \mathbb{C}^{g} / \Lambda$ et $B^{\prime} \simeq V /(\Lambda \cap V)$ pour un sous-espace vectoriel $V$ de $\mathbb{C}^{g},\left(A / B^{\prime}\right)^{\vee}$ s'identifie à $V^{\perp} /\left(V^{\perp} \cap \Lambda\right)$, où $V^{\perp}$ est l'orthogonal de $V$ dans $\mathbb{C}^{g}$ relativement à la forme de Riemann $H$ ). On en déduit aisément (voir par exemple [Be- $\mathrm{Ph}$, lemme 2 et prop. 3]), que $\operatorname{deg}\left(B^{\prime \perp}\right) \leq c_{55} \operatorname{deg}\left(B^{\prime}\right)$. En utilisant la relation $\operatorname{deg}(B)=\operatorname{deg}(\varphi) \operatorname{deg}\left(B^{\prime}\right)$ (voir [La2]), et l'inégalité $S \leq \operatorname{deg}(\varphi)$, la relation du lemme de zéros implique :

$$
T^{r+1} S \operatorname{deg}\left(B^{\prime}\right) \leq c_{50} L^{r+2},
$$

ce qui donne :

$$
S \operatorname{deg}\left(B^{\prime}\right) \leq c_{56} C_{0}^{16 g} \rho^{\operatorname{dim}\left(B^{\prime}\right)} .
$$

D'où l'on déduit aisément que $P$ est d'ordre $\leq c_{53} C_{0}^{32 g} \rho^{2 g}$ modulo $B^{\prime \perp}$, qui est, lui, de degré $\leq c_{52} C_{0}^{16 g} \rho^{g}$. Ce qui prouve le ThÉorème 1.4. On notera que l'on peut inclure dans la discussion générale les cas particuliers

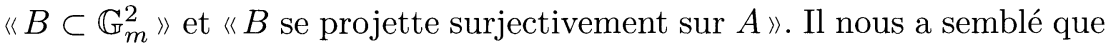
l'étude préalable de ces deux cas particuliers clarifiaient l'argument.

\section{BIBLIOGRAPHIE}

[Be] Bertrand (D.). - Endomorphismes de groupes algébriques; applications arithmétiques, Approximations diophantiennes et nombres transcendants, Progress in Math., 31, Birkhäuser, Boston-BaselStuttgart, 1983 . 
[Be-Ph] Bertrand (D.) et Philippon (P.). - Sous-groupes algébriques de groupes algébriques commutatifs, Illinois J. Math., t. 32 (2), 1988, p. 263-280.

[Dav1] David (S.). - Fonctions thêta et points de torsion des variétés abéliennes, Compositio Math., t. 78, 1991, p. 121-160.

[Dav2] David (S.). - Théorie de Baker dans les familles de groupes algébriques commutatifs, thèse de doctorat, Université de Paris VI, 1989 .

[De] Denis (L.). - Lemmes de multiplicités et intersections, C.R. Acad. Sci. Paris, t. 314, série I, 1992, p. 97-100.

[Fa-Wü] Faltings (G.) und Wüstholz (G.). - Einbettungen kommutativer algebraicher Gruppen und einige ihrer Eigenschaften, J. Reine Angew. Math., t. 354, 1984, p. 175-205.

[Hi-Si1] Hindry (M.) and Silverman (J.). - The canonical height and integer points on elliptic curves, Inv. Math., t. 93, 1988, p. 419-450.

[Hi-SI2] Hindry (M.) and Silverman (J.). - On Lehmer's conjecture for elliptic curves, [C. Goldstein éd.], Séminaire de théorie des nombres de Paris 1988-89, Progress in Math., Birkhäuser, Boston-BaselStuttgart, 1990, p. 103-116.

[Ig] Igusa (J.). - Theta functions. - Grundlehren Math. Wiss., 194, Springer, Berlin-Heidelberg-New York, 1972.

[Kem] Kempf (G.). - Multiplication over abelian varieties, Am. J. Math., t. 110, 1988, p. 765-773.

[Kn-La] Knop (F.) and LAnge (H.). - Commutative algebraic groups and intersection of quadrics, Math. Ann., t. 267, 1984, p. 555-571.

[L] LANG (S.). - Elliptic curves : diophantine analysis. - Grundlehen Math. Wiss., 231, Springer, Berlin-Heidelberg-New York, 1978.

[La1] LANGE (H.). - Families of translations of commutative algebraic groups, J. Algebra, t. 109, 1987, p. 260-265.

[La2] LAnge (H.). - A remark on the degrees of commutative algebraic groups, Illinois J. Math., t. 33 (3), 1989, p. 409-415.

[Lau] Laurent (M.). - Minoration de la hauteur de Néron-Tate, [M.-J. Bertin, éd.], Séminaire de théorie des nombres de Paris 1981-82, Progress in Math., Birkhäuser, Boston-Basel-Stuttgart, 1983, p. $137-152$.

[Ma1] Masser (D.). - Small values of heights on families of abelian varieties, Proc. Conf. Bonn, Lecture Notes 1290, Springer-Verlag 1985 , p. $109-148$.

[Ma2] Masser (D.). — Lettre à Daniel Bertrand du 17-11-86, nov. 1986.

[Ma3] Masser (D.). - Counting points of small height on elliptic curves, Bull. Soc. Math. France, t. 117, 1989, p. 247-265. 
[Ma4] MAsser (D.). — Lettre à l'auteur du 18-09-90, sept. 1990. Voir aussi Large periods matrices and a conjecture of Lang, à paraître dans le Séminaire de théorie des nombres 1991-92 [S. David, éd.], Progress in Math., Birkhäuser, Boston-Basel-Stuttgart, 1993, p. 153-177.

Ma-Wü] Masser (D.) and Wüstholz (G.). - Estimating isogenies on elliptic curves, Inv. Math., t. 100 (1), 1990, p. 1-24.

[M-B] Moret-Bailly (L.). - Compactifications, hauteurs et finitude, Séminaire sur les pinceaux arithmétiques : la conjecture de Mordell, L. Szpiro, Astérisque, 127, S.M.F. 1985, p. 113-129.

[Mum] Mumford (D.). - Abelian varieties, TIFR studies in mathematics, second edition. - Oxford University Press, 1974.

[M-Z] MANin (I.) and ZARHIN (G.).- Heights on families of abelian varieties, USSR Math. Sbornik, t. 18, 1972, p. 169-179.

[Ph] Philippon (P.). - Lemmes de zéros dans les groupes algébriques commutatifs, Bull. Soc. Math. France, t. 114, 1986, p. 355-383.

[P-W] Philippon (P.) et Waldschmidt (M.). - Formes linéaires de logarithmes dans les groupes algébriques commutatifs, Illinois J. Math., t. 32 (2), 1988, p. 281-314.

[Sh] Shimura (G.). - On the derivatives of theta functions and modular forms, Duke Math. J., t. 44, 1977, p. 365-387.

[Si1] Silverman (J.). - Lower bounds for the canonical height on elliptic curves, Duke Math. J., t. 48, 1981, p. 633-648.

[Si2] Silverman (J.). - Lower bounds for height functions, Duke Math. J., t. 51 (2), 1984, p. 395-403.

[Sz] SzPiro (L.). - Discriminant et conducteur des courbes elliptiques, Séminaire sur les pinceaux de courbes elliptiques, L. Szpiro, Astérisque, 183, S.M.F. 1990, p. 7-18.

[W] Waldschmidt (M.). - A lower bound for linear forms in logarithms, Acta Arith., t. 37, 1980, p. 257-283. 